\title{
THE STRUCTURE OF GROUP-LIKE EXTENSIONS OF MINIMAL SETS $\left(^{1}\right)$
}

\author{
BY \\ ROBERT ELLIS
}

Introduction. The aim of this paper is to develop further the algebraic theory of minimal sets begun in [6] and [7] and to apply it to obtain a structure theorem for the collection of group-like extensions of a given minimal set. When this minimal set is taken to be a single point, the resulting theorem reduces to the one obtained by Furstenberg [8].

The paper is divided into six sections. In $\$ 1$ and 2 the general algebraic theory is developed. Since I feel that this theory can be fruitfully applied to other problems in topological dynamics besides the one considered here, I have tried to make this exposition self-contained. Thus much of the discussion contained in these two sections is to be found in [6] and [7]. However, some of the notation has been changed for purposes of simplification. The new material, herein, is concerned with the construction and study of various topologies on a certain subgroup of the $\beta$-compactification of an arbitrary abstract group. These are the so called $\tau$ topologies of which brief mention was made in [7].

$\S 3$ is a collection of results from various papers inserted in order to make the overall exposition self-contained.

In $\$ 4$ the algebraic theory is applied to develop a structure theory of group-like extensions. The relation of this structure theory to that of Furstenberg for distal minimal sets [8] is exhibited. This is done by means of the notions of a principal extension and a principal bi-transformation group (analogous to a principal fiber bundle) introduced in this section.

The main proposition of this paper is 4.14 which is a general statement about principal group extensions. When applied to the situation at hand it yields a generalization of the Furstenberg results.

In $\$ 5$ the main proposition is applied to some special cases. Here the Bohr compactification of the abstract group $T$ is exhibited in terms of the algebraic theory previously described.

During the course of this paper and also in [6] and [7] various seemingly arbitrary choices are made. $\$ 6$ is devoted to showing that these choices are indeed natural.

1. In this first section I introduce some notation and definitions which will be used throughout the paper. A few basic lemmas are also proved.

Received by the editors June 29, 1967.

(1) This work was partially supported by the National Science Foundation under contract number GP-6325. 
1.1 Standing notation and definitions. In this paper $T$ will denote a group provided with its discrete topology, $\beta T$ its Stone-Čech compactification, and $\mathscr{C}=\mathscr{C}(\beta T)$ the Banach algebra of real valued continuous functions on $\beta T$ (with $\|f\|=\sup [|f(x)| / x \in \beta T]$ for all $f \in \mathscr{C})$.

Let $t \in T$. Then the map $R_{t}: T \rightarrow \beta T$ such that $R_{t}(s)=s t(s \in T)$ is continuous. Hence it has a continuous extension to all of $\beta T$, which I shall again denote by $R_{t}$.

Now let $x \in \beta T$. Then the map $L_{x}: T \rightarrow \beta T$, such that $L_{x}(t)=R_{t}(x)(t \in T)$ is continuous. (Recall that $T$ is discrete.) Hence $L_{x}$ may be extended to a continuous mapping (again denoted $L_{x}$ ) of $\beta T$ into $\beta T$.

For $x, y \in \beta T$, set $x y=L_{x}(y)$. Then $x t=L_{x}(t)=R_{t}(x)(x \in \beta T, t \in T)$, and it is natural to set $R_{y}(x)=x y(x, y \in \beta T)$. (Recall that $T$ may be viewed as a dense subset of $\beta T$.) In this way $\beta T$ is provided with a semigroup structure such that $L_{x}$ is continuous $(x \in \beta T)$ and $R_{x}$ is continuous $(x \in T)$.

Let $f \in \mathscr{C}, x \in \beta T$. Then it will be convenient to denote the image of $x$ under $f$ by $\langle f, x\rangle$.

Again let $f \in \mathscr{C}, x \in \beta T$. Then $f x$ will denote $L_{x}$ followed by $f$ and $x f$ will denote $R_{x}$ followed by $f$. Thus $\langle f x, y\rangle=\langle f, x y\rangle$ and $\langle x f, y\rangle=\langle f, y x\rangle(f \in \mathscr{C}, x, y \in \beta T)$. Note that $f x \in \mathscr{C}(f \in \mathscr{C}, x \in \beta T)$, whereas all that can be said in general about $x f$ is that it is in $\mathscr{C}$ when $f \in \mathscr{C}$ and $x \in T$.

By a $T$-subalgebra $\mathscr{A}$ of $\mathscr{C}$ I shall mean a uniformly closed subalgebra of $\mathscr{C}$ containing the constant functions and such that $t f \in \mathscr{A}(t \in T, f \in \mathscr{A})$.

One may view $\mathscr{C}$ as the set of continuous real valued functions on $\beta T$ or alternatively as the collection of bounded real valued functions on $T$. This latter point of view is useful in defining elements of $\mathscr{C}$. Thus let $K \subset \beta T, f \in \mathscr{C}$. Then $f^{K},\left(f_{K}\right)$, will denote that element of $\mathscr{C}$ such that $\left\langle f^{K}, t\right\rangle=\sup [\langle f x, t\rangle \mid x \in K]\left(\left\langle f_{K}, t\right\rangle=\right.$ $\inf [\langle f x, t\rangle \mid x \in K]$ ) for all $t \in T$. (This notation does not agree with that of [6] but is more convenient.) The reader is cautioned that in general $\left\langle f^{K}, y\right\rangle$ need not equal sup $[\langle f x, y\rangle \mid x \in K]$ nor need $\left\langle f_{K}, y\right\rangle$ equal inf $[\langle f x, y\rangle \mid x \in K]$ when $y$ is an arbitrary element of $\beta T$. One deduces from the continuity of $f^{K}$ and $f_{K}$, that $\left\langle f^{K}, y\right\rangle$ $\geqq \sup [\langle f, x y\rangle \mid x \in K]$ and that $\left\langle f_{K}, y\right\rangle \leqq \inf [\langle f, x y\rangle \mid x \in K]$ for all $y \in \beta T$.

1.2 Lemma. The following statements hold for all $f \in \mathscr{C}, x \in \beta T, t \in T$, and nonvacuous subsets $K \subset \beta T$.

1. $(-f)^{R}=-\left(f_{E}\right)$,

2. $(t f)^{K}=t\left(f^{K}\right)$,

$2^{\prime} . \quad(t f)_{E}=t\left(f_{B}\right)$,

3. $(f x)^{K}=f^{x x}$,

3'. $(f x)_{K}=f_{x K}$,

4. $f^{K x} \leqq f^{K} x$,

$4^{\prime} . \quad f_{K} x \leqq f_{K x}$. 
Proof. Let me first remark that $2^{\prime}, 3^{\prime}$, and $4^{\prime}$ follow from 1 together with 2,3 , and 4 respectively. Hence I shall only prove $1,2,3$, and 4 .

1 .

$$
\begin{aligned}
\left\langle(-f)^{K}, s\right\rangle & =\sup [\langle-f, y s\rangle \mid y \in K] \\
& =\sup [-\langle f, y s\rangle \mid y \in K]=-\inf [\langle f, y s\rangle \mid y \in K] \\
& =-\left\langle f_{K}, s\right\rangle \quad(s \in T) . \\
\left\langle(t f)^{K}, s\right\rangle & =\sup [\langle t f, y s\rangle \mid y \in K] \\
& =\sup [\langle f, y s t\rangle \mid y \in K]=\left\langle f^{K}, s t\right\rangle \\
& =\left\langle t f^{K}, s\right\rangle \quad(s \in T) .
\end{aligned}
$$

3. Let $x \in \beta T$. Then for all $t \in T$,

$$
\begin{aligned}
\left\langle(f x)^{K}, t\right\rangle & =\sup [\langle f x, y t\rangle \mid y \in K]=\sup [\langle f, x y t\rangle \mid y \in K] \\
& =\sup [\langle f, z t\rangle \mid z \in x K]=\left\langle f^{x K}, t\right\rangle .
\end{aligned}
$$

4. Let $s \in T, z \in K x$. Then there exists $y \in K$ with $z=y x$. Since $y \in K, f y \leqq f^{K}$. Hence $\langle f z, s\rangle=\langle f y x, s\rangle=\langle f y, x s\rangle \leqq\left\langle f^{K} x, s\right\rangle$. This implies that $f^{K x} \leqq f^{K} x$.

1.3 Lemma. Let $f, g \in \mathscr{C}, K, L$ nonvacuous subsets of $\beta T, h=f \wedge g$. Then $h^{K \cup L}$ $\leqq \min \left(f^{K} \vee g^{L}, f^{L} \vee g^{K}\right) ; \quad$ (where if $F, G \in \mathscr{C}, F \wedge G=\inf (F, G)$ and $F \vee G=$ $\sup (F, G))$.

Proof. By definition $\left\langle h^{K \cup L}, t\right\rangle=\sup [\langle h a, t\rangle \mid a \in K \cup L]$. Now $\langle h a, t\rangle \leqq\langle f a, t\rangle$ $\leqq\left\langle f^{K}, t\right\rangle$ if $a \in K$ and $\langle h a, t\rangle \leqq\langle g a, t\rangle \leqq\left\langle g^{L}, t\right\rangle$ if $a \in L$. Thus in either case $\langle h a, t\rangle$ $\leqq\left\langle f^{K} \vee g^{L}, t\right\rangle$. Since this holds for all $t \in T$ and all $a \in K \cup L$, we may conclude that $h^{K \cup L} \leqq f^{K} \vee g^{L}$. Similarly $h^{K \cup L} \leqq f^{L} \vee g^{K}$.

2. The semigroup structure on $\beta T$ defined in $\S 1$ induces an action of $T$ on $\beta T$ so that the pair $(\beta T, T)$ becomes a transformation group in the sense of [9]. Since $\beta T$ is compact, [9] guarantees the existence of a minimal subset $M$ of $\beta T$. This means that $M T \subset M$ and that $\mathrm{cl}(x T)=M(x \in M)$. The transformation group $(M, T)$ is universal in the sense that if $(X, T)$ is any minimal transformation group, where $X$ is compact Hausdorff, then there exists a homomorphism of $(M, T)$ onto $(X, T)$; i.e. a continuous mapping $\pi$ of $M$ onto $X$ such that $\pi(m t)=\pi(m) t(m \in M, t \in T)$. (See [5] for details.)

In general there are many minimal subsets of $\beta T$, but they are all isomorphic [5]. One can also describe the minimal subsets of $\beta T$ in terms of the semigroup structure; namely they are just the minimal right ideals of $\beta T$ [4].

As in [7] let $M$ be some minimal subset of $\beta T$ and $u$ a fixed idempotent in $M$. (Such exists by [4].) Then the semigroup structure on $\beta T$ induces a group structure on $G=M u[4]$.

In this section I shall define and study various topologies on $G$ and subgroups of $G$ : These will include the $\tau$-topology introduced in [7]. 
Although $M$ and $u$ are chosen arbitrarily, it will be shown later that the.results obtained are "independent" of the choices made.

2.1 Standing notation. Henceforth $M$ will denote a fixed minimal subset of $\beta T$, $u$ a fixed idempotent thereof, and $G$ the group $M u$. As in [7] I shall denote the elements of $G$ by lower case Greek letters, $\omega$ designating the element $u$.

Let $K \subset G, f \in \mathscr{C}$. Define

and

$$
\begin{aligned}
& K^{f}=\left[\alpha \mid \alpha \in G \text { and } f \alpha \leqq f^{K} \omega\right], \\
& K_{f}=\left[\alpha \mid \alpha \in G \text { and } f_{K} \omega \leqq f \alpha\right],
\end{aligned}
$$

$$
K(f)=K^{f} \cap K_{f}
$$

It follows from 1 of Lemma 1.2 that $K^{f}=K_{-f}$ for all subsets $K$ of $G$ and element $f$ of $\mathscr{C}$.

2.2 Proposition. Let $\mathscr{A}$ be a $T$-subalgebra of $\mathscr{C}$. Then the mapping $K \rightarrow \operatorname{cls}_{\mathscr{A}} K$ $=\bigcap[K(f) \mid f \in \mathscr{A}]$, from subsets of $G$ to subsets of $G$ defines a closure operator on $G$.

Before proceeding with the proof let me remark that since $\mathscr{A}$ is an algebra,

$$
\begin{aligned}
\operatorname{cls}_{\mathscr{A}} K & =\bigcap\left[K^{f} \cap K_{f} \mid f \in \mathscr{A}\right]=\bigcap\left[K^{f} \cap K^{-f} \mid f \in \mathscr{A}\right] \\
& =\bigcap\left[K^{f} \mid f \in \mathscr{A}\right]=\bigcap\left[K_{-f} \mid f \in \mathscr{A}\right]=\bigcap\left[K_{f} \mid f \in \mathscr{A}\right] .
\end{aligned}
$$

Proof. The proof is essentially the same as that in [7] but I include it for completeness sake.

Let $K$ and $L$ be arbitrary subsets of $G$. Then we must verify that

(I) $K \subset \operatorname{cls}_{\mathscr{A}} K$;

(II) if $K \subset L$, then $\operatorname{cls}_{\mathscr{A}} K \subset \mathrm{cls}_{\mathscr{A}} L$;

(III) $\operatorname{cls}_{\mathscr{A}}\left(\operatorname{cls}_{\mathscr{A}} K\right)=\operatorname{cls}_{\mathscr{A}} K$; and

(IV) $\operatorname{cls}_{\mathscr{A}}(K \cup L)=\operatorname{cls}_{\mathscr{A}} K \cup \mathrm{cls}_{\mathscr{A}} L$.

With regard to (IV) note that (I) and (II) imply that cls $K \cup \operatorname{cls} L \subset \operatorname{cls}(K \cup L)$. Now suppose that $\alpha \notin$ cls $K \cup$ cls $L$. Then there exist $f, g \in \mathscr{A}$ such that $\alpha \notin K^{f} \cup L^{g}$. This means that there exist $t, s \in T$ such that $\langle f, \alpha t\rangle\rangle\left\langle f^{R}, \omega t\right\rangle$ and $\langle g, \alpha s\rangle$ \rangle$\left\langle g^{L}, \omega s\right\rangle$. Since $\mathscr{A}$ contains the constant functions, we may assume that $\langle f, \alpha t\rangle$ $=\langle g, \alpha s\rangle$. Since $\mathscr{A}$ is a $T$-subalgebra, it contains the function $h=t f \wedge s g$. Then

$$
\begin{aligned}
\langle h, \alpha\rangle & =\langle f, \alpha t\rangle=\langle g, \alpha s\rangle>\max \left(\left\langle(t f)^{K}, \omega\right\rangle,\left\langle(s g)^{L}, \omega\right\rangle\right) \\
& \left.=\dot{\langle}(t f)^{K} \vee(s g)^{L}, \omega\right\rangle \geqq\left\langle h^{K \cup L}, \omega\right\rangle
\end{aligned}
$$

by Lemma 1.3. Hence $\alpha \notin(K \cup L)^{h}$ whence $\alpha \notin$ cls $K \cup L$. The proof is completed.

If $\mathscr{A}$ is a $T$-subalgebra of $\mathscr{C}$, then $\tau(A)$ will denote the topology induced on $G$ by the above closure operator. Thus the $\tau$-topology introduced in [7] coincides with $\tau(\mathscr{C})$.

If $K \subset \beta T$, then $\bar{K}$ will denote the ordinary closure of $K$ in $\beta T$. Thus if $K \subset G$, and $\mathscr{A}$ is a $T$-subalgebra of $\mathscr{C}$, cls $_{\mathscr{A}} K \subset G$ whereas $\bar{K}$ is in general not contained in $G$. (Of course in this case $\bar{K} \subset M$.) 


\subsection{REMARKS.}

1. One might try to define a closure operator on all of $\beta T$ by dropping the restriction that $K$ be a subset of $G$. However, the resulting function from subsets of $\beta T$ to subsets of $\beta T$ is not a closure operator. (The operator so defined is not idempotent.) The difficulty arises from the presence of the idempotent $\omega$ in the definition of $K^{f}$. If one eliminates this difficulty i.e. defines cls $K=[x \mid x \in \beta T$ and $\left.f x \leqq f^{K}(f \in \mathscr{C})\right]$, then one obtains the ordinary topology on $\beta T$. Let me indicate the proof of the above fact since I will use it later.

Let $K \subset \beta T, x \in \bar{K}, f \in \mathscr{C}$, and $t \in T$. Then there exists a net $k_{n}$ in $K$ with $k_{n} \rightarrow x$. Hence $\left\langle f, k_{n} t\right\rangle=\left\langle t f, k_{n}\right\rangle \rightarrow\langle t f, x\rangle=\langle f, x t\rangle$. This implies that $\left\langle f^{K}, t\right\rangle \geqq\langle f, x t\rangle$; whence $f x \leqq f^{K}$.

On the other hand, if $x \notin \bar{K}$ then there exists $f \in \mathscr{C}$ with $\langle f, x\rangle=1$ and $\langle f, y\rangle=0$ $(y \in \bar{K})$. This implies that $f x \leq f^{K}$.

2. Let $\mathscr{A}$ and $\mathscr{B}$ be two $T$-subalgebras of $\mathscr{C}$ with $\mathscr{A} \subset \mathscr{B}$. Then it is immediate from the definition that $\tau(\mathscr{A}) \subset \tau(\mathscr{B})$.

3. By Lemma 1.2 number $3,(f \omega)^{K}=f^{\omega K}(f \in \mathscr{C}, K \subset \beta T)$. Hence if $K \subset G$, $\omega K=K$ and $(f \omega)^{K}=f^{K}(f \in \mathscr{C})$. Of course $(f \omega) \alpha=f \alpha(\alpha \in G, f \in \mathscr{C})$. Thus we may conclude that $\alpha \in K^{f}$ if and only if $\alpha \in K^{f \omega}$. This implies that $\tau(\mathscr{A})=\tau(\mathscr{A} \omega)$ for all $T$-subalgebras, $\mathscr{A}$ of $\mathscr{C}$. Thus in defining the various $\tau$-topologies on $G$ we may confine our attention to those $T$-subalgebras which are contained in $\mathscr{M}=[f \mid f \in \mathscr{C}$ and $f \omega=f]$ (note that $\omega^{2}=\omega$ implies that $\mathscr{A} \omega \subset \mathscr{M}$ ). In particular the above considerations show that $\tau(\mathscr{C})=\tau(\mathscr{M})$. ( $\mathscr{M}$ is nothing but $\mathscr{C} \omega$.)

2.4 LEMMA. Let $K \subset G, x \in \bar{K}$. Then $x \omega \in \mathrm{cls}_{\mathscr{C}} K$.

Proof. Let $f \in \mathscr{C}$. Then by $2.3,1, f x \leqq f^{K}$. Hence $f x \omega \leqq f^{K} \omega$ and so $x \omega \in K^{f}$.

2.5 Proposition. $(G, \tau(\mathscr{C}))$ is a compact, $T_{1}$ space.

Proof. Let $\left(K_{i} \mid i \in I\right)$ be a family of $\tau(\mathscr{C})$-closed subsets of $G$ with the finite intersection property. Then $\left(\bar{K}_{i} \mid i \in I\right)$ is a family of closed subsets of $\beta T$, again with the finite intersection property. Since $\beta T$ is compact, there exists $x \in \bar{K}_{i}(i \in I)$. By $2.4 x \omega \in$ cls $_{\mathscr{C}} K_{i}=K_{i}(i \in I)$. Thus $(G, \tau(G))$ is compact.

Now let $\alpha, \beta \in G$ with $\alpha \neq \beta$. Then there exists $f \in \mathscr{C}$ with $\langle f, \alpha\rangle\langle\langle f, \beta\rangle$. Hence $\beta \notin \alpha^{f}$ and so $\beta \notin \operatorname{cls}_{\mathscr{G}} \alpha$. Thus $\alpha=\operatorname{cls}_{\mathscr{G}} \alpha$.

2.6 Proposition. Let $\mathscr{A}$ be a $T$-subalgebra of $\mathscr{C}$. Then

(i) the map $R_{\alpha}:(G, \tau(\mathscr{A})) \rightarrow(G, \tau(\mathscr{A}))$ is continuous for all $\alpha \in G$;

(ii) the map $L_{\alpha}:(G, \tau(\mathscr{A})) \rightarrow(G, \tau(\mathscr{A}))$ is continuous for all $\alpha \in G$ such that $\mathscr{A} \alpha \subset \mathscr{A}$;

(iii) the map $\beta \rightarrow \beta^{-1}$ of $(G, \tau(\mathscr{C}))$ into $(G, \tau(\mathscr{C}))$ is continuous.

Proof. (i) Let $K \subset G, \beta \in \operatorname{cls}_{\mathscr{A}} K, f \in \mathscr{A}$. Then from $f \beta \leqq f^{K} \omega$ we deduce that $f \beta \alpha \leqq f^{K} \omega \alpha=f^{K} \alpha$. Now $K=K \omega=K \alpha \alpha^{-1}$, whence $f^{K} \alpha=f^{K \alpha \alpha-1} \alpha \leqq f^{K \alpha} \alpha^{-1} \alpha=f^{K \alpha}$; 
by Lemma 1.2, 4. Thus $\beta \alpha \in(K \alpha)^{\prime}$. Since $f$ was arbitrary, we have shown that $R_{\alpha}\left(\operatorname{cls}_{\mathscr{A}} K\right) \subset \mathrm{cls}_{\mathscr{A}} R_{\alpha}(K)$. Hence $R_{\alpha}$ is continuous.

(ii) Let $K \subset G, \beta \in \operatorname{cls}_{\mathscr{A}} K, f \in \mathscr{A}$ and $\alpha \in G$ with $\mathscr{A} \alpha \subset \mathscr{A}$. Then $f \alpha \in \mathscr{A}$, whence $f \alpha \beta \leqq(f \alpha)^{K} \omega=f^{\alpha K} \omega$; i.e. $\alpha \beta \in(\alpha K)^{\prime}$. Thus $L_{\alpha}\left(\operatorname{cls}_{\mathscr{A}} K\right) \subset \operatorname{cls}_{\mathscr{A}} L_{\alpha} K$, whence $L_{\alpha}$ is continuous.

(iii) Let $K \subset G, \beta \in$ cls\& $K, f \in \mathscr{C}$. I shall show that $\beta^{-1} \in\left(K^{-1}\right)^{f}$ which will imply the continuity of the inverse since $K$ and $f$ are arbitrary.

Set $h=f^{K^{-1}}$. Then $h \in \mathscr{C}$ and $h_{K} \omega \leqq h \beta$ since $\beta \in K_{h}$. Let $t \in T$. Then $\left\langle h_{K}, t\right\rangle$ $=\inf [\langle h, \alpha t\rangle \mid \alpha \in K]$. Let $\alpha \in K$. Then $\langle h, \alpha t\rangle=\left\langle f^{K^{-1}}, \alpha t\right\rangle \geqq \sup \left[\left\langle f, \gamma^{-1} \alpha t\right\rangle \mid \gamma \in K\right]$ $\geqq\langle f, t\rangle$ since $\alpha \in K$. Hence $\langle h, \alpha t\rangle \geqq\langle f, t\rangle(\alpha \in K)$, whence $\left\langle h_{K}, t\right\rangle \geqq\langle f, t\rangle$. Thus $h_{K} \geqq f$ and so $f^{K^{-1}} \beta=h \beta \geqq h_{K} \omega \geqq f \omega$. This implies that $f \beta^{-1} \leqq f^{K^{-1}} \omega ;$; i.e. $\beta^{-1}$ $\in\left(K^{-1}\right)^{f}$.

It will be convenient for expository purposes to abstract the situation described in Propositions 2.5 and 2.6. Thus for the remainder of this section $S$ will denote a group provided with a topology with respect to which $S$ is compact and such that multiplication is continuous in each variable separately. (Note: I assume no separation axioms nor that inversion is continuous.) Let $\mathscr{N}$ denote the neighborhood filter at the identity $e$ of $S$ and $Q$ the intersection of the closures of the elements of $\mathcal{N}$, i.e. $Q=\cap[\bar{V} \mid V \in \mathscr{N}]$. I shall show that $Q$ is a closed normal subgroup of $S$ such that $S / Q$ is a compact Hausdorff topological group.

\subsection{Lemma. Let $\phi \neq A \subset S$. Then $\bar{A}=\bigcap\left[A V^{-1} \mid V \in \mathscr{N}\right]$.}

Proof. The standard "topological group proof" works equally well here.

\subsection{LEMMA. $Q$ is a closed invariant subgroup of $S$.}

Proof. That $Q$ is closed follows from its definition as the intersection of closed sets. Now let $x, y \in Q, V, W \in \mathcal{N}$. Since $y \in \operatorname{cl}$ (int $V$ ), $y W \cap$ int $V \neq \varnothing$. Let $a \in W$ with $y a \in$ int $V$. Then there exists $U \in \mathscr{N}$ with $U y a \subset V$, whence $\bar{U} y a \subset \bar{V}$ (recall that right multiplication by $y a$ is a homeomorphism of $S$ onto $S$ ). Now $x \in \bar{U}$. Then $x y a \in \bar{V}$ and $x y \in \bar{V} W^{-1}$. Since $W$ is arbitrary, $x y \in \operatorname{cl}(\mathrm{cl}(V))=\bar{V}$ by Lemma 2.7. Thus $x y \in \cap[\bar{V} \mid V \in \mathscr{N}]=Q$.

Now let $x \in Q$. Set $\mathscr{E}=[E \mid E$ is a nonvacuous closed subset of $S$ such that $E^{2} \subset E \subset x Q$ ]. Since $(x Q)(x Q) \subset x Q(Q Q) \subset x Q^{2} \subset x Q, x Q \in \mathscr{E}$. The compactness of $S$ allows one to conclude immediately that $\mathscr{E}$ is inductive when ordered by inclusion. Let $E$ be a minimal element of $\mathscr{E}$ and $u \in E$. Then $(u E)(u E) \subset u E\left(E^{2}\right)$ $\subset u E^{2} \subset u E \subset E^{2} \subset E \subset x Q$. Moreover, since $\varnothing \neq u E$ and $u E$ is closed, $u E \in \mathscr{E}$. The minimality of $E$ implies that $u E=E$. Since $u \in E$, this means that $u y=u$ for some $y \in E$. Since $S$ is a group, $y=e$; i.e. $e \in E \subset x Q$. Hence $x^{-1} \in Q$.

Finally let $a \in S, V \in \mathscr{N}$. Set $f(x)=a x a^{-1}(x \in S)$. Then $f$ is a continuous mapping of $S$ into $S$ with $f(e)=e$. Hence there exists $W \in \mathscr{N}$ with $f(W) \subset V$. The continuity of $f$ implies that $f(\bar{W}) \subset \bar{V}$. Thus $f(Q) \subset \bar{V}$. Since $V$ was arbitrary, $f(Q) \subset Q$; i.e. $a Q a^{-1} \subset Q$. The proof is completed. 
2.9 Lemma. Let $V$ be an open element of $\mathcal{N}$. Then $Q \bar{V}=\bar{V}$.

Proof. Let $y \in V$. Since $V$ is open, there exists $W \in \mathscr{N}$ with $W y \subset V$. This implies $\bar{W} y \subset \bar{V}$. Now $Q \subset \bar{W}$. Hence $Q y \subset \bar{V}(y \in V)$, i.e. $Q V \subset \bar{V}$. Let $x \in Q$. Then $x V \subset \bar{V}$ implies that $x \bar{V} \subset \bar{V}$. Thus $Q \bar{V} \subset \bar{V}$.

2.10 Proposition. $S / Q$ is a compact Hausdorff topological group. Moreover, if $H$ is a subgroup of $S$ then the coset space $S / H=[H x \mid x \in S]$ is Hausdorff if and only if $H$ is closed and $H \supset Q$.

(Note: The above statement applies equally well to the coset space $[x H \mid x \in S]$.

Proof. Let $\Pi: S \rightarrow S / Q$ be the canonical map, $A \subset S$. Then first I would like to show that $\Pi^{-1} \Pi(A)=Q A$. Now $x \in \Pi^{-1} \Pi(A)$ if and only if $\Pi(x)=\Pi(a)$ for some $a \in A$. This last can happen if and only if $x a^{-1} \in Q$, i.e. $x \in Q A$.

Let $x, y \in S$ with $\Pi(x) \neq \Pi(y)$. Then $x y^{-1} \notin Q$. Thus there exists an open element $V$ of $\mathscr{N}$ with $x y^{-1} \notin \bar{V}$. This implies the existence of an open element $W$ of $\mathscr{N}$ with $W x y^{-1} \cap \bar{V}=\varnothing$. By Lemma $2.9 Q \bar{V}=\bar{V}$. Thus $W x y^{-1} \cap Q V=\varnothing$. Hence $Q W x \cap Q V y=\varnothing$. Now $Q W x$ and $Q V y$ are open subsets of $S$ with $\Pi^{-1} \Pi(W x)$ $=Q W x$ and $\Pi^{-1} \Pi(V y)=Q V y$. Thus $\Pi(W x)$ and $\Pi(W y)$ are nonintersecting neighborhoods of $\Pi(x)$ and $\Pi(y)$ respectively.

Let $x \in S$. To show that the map $y Q \rightarrow x y Q(y \in S)$ of $S / Q$ into $S / Q$ is continuous it suffices to show that its composite with $\Pi$ is continuous. But this composition is merely $\Pi$ composed with left multiplication by $x$ on $S$. Similarly, the map $y Q \rightarrow y x Q(y \in S)$ of $S / Q$ into $S / Q$ is continuous. Hence $S / Q$ is a topological group by [2].

Now suppose $H$ is a subgroup of $S$ such that $S / H$ is Hausdorff. Since $H$ is the inverse image of $\{H\}$ under the canonical map $\rho: S \rightarrow S / H, H$ is a closed subset of $S$. Let $N$ be a closed neighborhood of $\{H\}$ in $S / H$. Then there exists $V \in \mathscr{N}$ with $\rho(V) \subset N$. Since $N$ is closed, this implies that $\rho(\bar{V}) \subset N$, whence $\rho(Q) \subset N$. Since $S / H$ is Hausdorff, the intersection of the closed neighborhoods of $\{H\}$ is just $\{H\}$ itself. Thus $\rho(Q)=\{H\}$ and therefore, $Q \subset H$.

Finally, let $H$ be a closed subgroup of $S$ with $Q \subset H$. Then $\Pi(H)$ is a closed subgroup of $S / Q$ with $S / H$ homeomorphic to $(S / Q) / \Pi(H)$.

2.11 Notation. Let $H$ be a subgroup of $G, \mathscr{A}$ a $T$-subalgebra of $\mathscr{C}$. Then $Q(H, \mathscr{A})$ will denote $\cap\left[\operatorname{cls}_{\mathscr{A}} V \mid V\right.$ a $\tau(\mathscr{A})$-neighborhood in $H$ of $\left.\omega\right]$. When there is no possibility of confusion, this set will be denoted $Q(\mathscr{A})$ or simply $Q$.

2.12 Proposition. Let $\mathrm{H}$ be a $\tau(\mathscr{C})$-closed subgroup of $G$ and $\mathscr{A}$ a $T$-subalgebra of $\mathscr{C}$ such that $\mathscr{A} H \subset \mathscr{A}$. Then $H / Q(H, \mathscr{A})$ is a compact Hausdorff topological group when provided with the quotient topology induced by the topology $\tau(\mathscr{A})$ on $H$.

Proof. Since $(G, \tau(\mathscr{C}))$ is compact by Proposition 2.5 and $H$ is $\tau(\mathscr{C})$-closed, $(H, \tau(\mathscr{C}))$ is compact. Now $\tau(\mathscr{A}) \subset \tau(\mathscr{C})$ implies that $(H, \tau(\mathscr{A}))$ is compact. Finally 
the fact that $\mathscr{A} H \subset \mathscr{A}$ together with (i) and (ii) of Proposition 2.6 allows us to apply Proposition 2.10 to obtain the desired conclusion.

3. In the rest of this paper I would like to apply the machinery developed in $§ 2$ to questions in topological dynamics. In particular I wish to reprove Furstenberg's structure theorem for minimal distal transformation groups [8] and indeed to generalize it to include the class of grouplike extensions introduced in [7].

In this section I shall recall some notions and results from other papers in order to make this exposition more or less self-contained.

Let $\mathscr{A}$ be a $T$-subalgebra of $\mathscr{C}$. Then a $T$-homomorphism $\phi$ of $\mathscr{A}$ into $\mathscr{C}$ is an algebra homomorphism of $\mathscr{A}$ into $\mathscr{C}$ such that $\phi(t f)=t \phi(f)(t \in T, f \in \mathscr{A})$. The set of $T$-homomorphisms of $\mathscr{A}$ into $\mathscr{C}$ will be denoted by $|\mathscr{A}|$.

The set $|\mathscr{A}|$ is given the structure of a transformation group as follows: First $|\mathscr{A}|$ is provided with the smallest topology making the real valued function $\phi \rightarrow\langle f \phi, e\rangle$ $(\phi \in|\mathscr{A}|)$ continuous for all $f \in \mathscr{A}$. This topology makes $|\mathscr{A}|$ into a compact Hausdorff space. Then $T$ is allowed to act on $|\mathscr{A}|$ via the map $(\phi, t) \rightarrow \phi t(\phi \in|\mathscr{A}|$, $t \in T)$, where $\phi t$ is that element of $|\mathscr{A}|$ such that $(\phi t)(f)=\phi(f) t$. It is easily verified that this action makes the pair $(|\mathscr{A}|, T)$ a transformation group. If moreover $\mathscr{A} \subset \mathscr{M}$, (the algebra associated with the minimal subset $M$ of $\beta T$ ) then $(|\mathscr{A}|, T)$ is a minimal set; i.e. the orbit $[\phi t / t \in T]$ is dense in $|\mathscr{A}|$ for all $\phi \in|\mathscr{A}|$.

As in [6] I shall say that the $T$-subalgebra $\mathscr{A}$ of $\mathscr{C}$ has a certain recursive property if the corresponding transformation group $(|\mathscr{A}|, T)$ possesses that property. Thus, for example, I shall say that $\mathscr{A}$ is minimal if $(|\mathscr{A}|, T)$ is minimal.

The above construction gives rise to a large class of transformation groups as the following proposition reveals.

3.1 Proposition. [6, Proposition 3]. Let $(X, T)$ be a minimal set with $X$ compact Hausdorff. Then there exists a T-subalgebra $\mathscr{A}$ of $\mathscr{C}$ such that $\mathscr{A} \subset \mathscr{M}$ and $(|\mathscr{A}|, T)$ is isomorphic to $(X, T)$.

Thus we can study the class of minimal sets with compact Hausdorff phase spaces by studying the collection of $T$-subalgebras of $\mathscr{M}$.

Given a $T$-subalgebra $\mathscr{A}$ of $\mathscr{C}$ it is desirable to know what the elements of $|\mathscr{A}|$ "look like". To this end let $\hat{p}$ denote the map $f \rightarrow f p(f \in \mathscr{C})$ of $\mathscr{C}$ into $\mathscr{C}$, where $p$ is an arbitrary element of $\beta T$. Then it is easily verified that $\hat{p} \in|\mathscr{C}|$.

The set $|\mathscr{C}|$ can be made into a semigroup by defining $\phi \psi$ for $\phi, \psi \in|\mathscr{C}|$ to be that element of $|\mathscr{C}|$ such that $f(\phi \psi)=(f \phi) \psi(f \in \mathscr{C})$.

3.2 Proposition [6, Lemma 2 and Corollary 1 of Proposition 1].

1. The map $p \rightarrow \hat{p}$ is a semigroup and a transformation group isomorphism of $\beta T$ onto $|\mathscr{C}|$.

2. Let $\mathscr{A}$ be a $T$-subalgebra of $\mathscr{C}$ and $\phi \in|\mathscr{A}|$.

Then there exists $p \in \beta T$ such that $\phi=\hat{p} \mid \mathscr{A}$. 
Because of 3.2 I shall identify $\beta T$ with $|\mathscr{C}|$ and use the same letter to denote an element of $\beta T$ and $|\mathscr{C}|$. Thus $p \in \beta T$ will also denote the map $f \rightarrow f p(f \in \mathscr{C})$ of $\mathscr{C}$ into $\mathscr{C}$. Moreover if $\mathscr{A}$ is a $T$-subalgebra of $\mathscr{C}$ and $p \in \beta T$ then $p \mid \mathscr{A}$ will denote the restriction to $\mathscr{A}$ of the map $f \rightarrow f p(f \in \mathscr{C})$. In fact when there is no chance of confusion I shall denote $p \mid \mathscr{A}$ by $p$ and thus view $p$ as an element of $|\mathscr{A}|$.

3.3 Notation. Let $\mathscr{A}$ be a $T$-subalgebra of $\mathscr{M}$. Then $\mathscr{S}(\mathscr{A})$ will denote the suibset $[\alpha \mid f \alpha=f,(f \in \mathscr{A})]$ of $G$.

Let $H$ be a subset of $G$. Then $\mathfrak{U}(H)$ will denote the subset $[f \mid f \alpha=f,(\alpha \in H)]$ of $\mathscr{C}$.

It is shown in [7] that $\mathbb{S}(\mathscr{A})$ is a $\tau(\mathscr{C})$-closed subgroup of $G$. Moreover, if $H$ is a $\tau(\mathscr{C})$-closed subgroup of $G$, then $\mathfrak{A}(H)$ is a $T$-subalgebra of $\mathscr{C}$ such that $\mathfrak{A}(H) \subset \mathscr{M}$ and $\operatorname{sir}(H)=H$.

3.4 Definition. Let $\mathscr{A}$ be a $T$-subalgebra of $\mathscr{C}$. Then $\mathscr{A}$ is distal if $f p w=f p$ for all $f \in \mathscr{A}, p \in \beta T$, and idempotents $w$ in $M$. (This is equivalent to requiring that $(|\mathscr{A}|, T)$ be distal in the sense of [9]; see [11] and [3].)

3.5 Definition [7]. Let $\mathscr{A}, \mathscr{B}$ be $T$-subalgebras of $\mathscr{C}$ with $\mathscr{A} \subset \mathscr{B} \subset \mathscr{M}$. Then $\mathscr{B}$ is a group-like extension of $\mathscr{A}$ if $p w|\mathscr{A}=p| \mathscr{A}$ implies that $p w|\mathscr{B}=p| \mathscr{B}$ for all $p \in \beta T$ and all idempotents $w$ in $M$. If in addition, $\mathscr{S}(\mathscr{B})$ is a normal subgroup of $\mathscr{S}(\mathscr{A})$, then $\mathscr{B}$ is called a group extension of $\mathscr{A}$.

3.6 Definition. Let $(X, T)$ and $(H, X)$ be transformation groups with the same phase space $X$. If the elements of $H$ commute with those of $T$, then the pair of transformation groups $\{(X, T),(H, X)\}$ is called a bitransformation group and is denoted $(H, X, T)$. In such a situation the orbit space $X / H=[H x / x \in X]$ becomes a transformation group with phase group $T$ in a natural way.

The following propositions relate the various concepts introduced above.

3.7 Proposition [7, Proposition 20]. Let $\mathscr{A}$ be a T-subalgebra of $\mathscr{M}$. Then $\mathscr{A}$ is distal if and only if $\mathscr{A}$ is a group-like extension of the algebra of constant functions, $R$.

3.8 Proposition [7, Proposition 27]. Let $\mathscr{A}$ and $\mathscr{B}$ be T-subalgebras of $\mathscr{M}$ such that $\mathscr{A} \subset \mathscr{B}$. Then $\mathscr{B}$ is a group extension of $\mathscr{A}$ if and only if there is a group of homeomorphisms $H$ of $|\mathscr{B}|$ onto $|\mathscr{B}|$ such that $(H,|\mathscr{B}|, T)$ is a bitransformation group and $(|\mathscr{B}| / H, T)$ is isomorphic to $(|\mathscr{A}|, T)$. In this case $H$ is isomorphic to $\mathbb{S}(\mathscr{A}) / \mathscr{S}(\mathscr{B})$.

In [7] a "neo-Galois theory" was developed in the context of group-like extensions. See in particular Proposition 19 of [7]. The following generalization of that proposition has been proved by Horelick [10].

3.9 Proposition [10]. Let $\mathscr{A}, \mathscr{B}$ be $T$-subalgebras of $\mathscr{M}$ such that $\mathscr{B}$ is a group-like extension of $\mathscr{A}$, let $\boldsymbol{F}=[\mathscr{F} \mid \mathscr{F}$ is a T-subalgebra of $\mathscr{C}$ with $\mathscr{A} \subset \mathscr{F} \subset \mathscr{B}]$, and let $\boldsymbol{G}=[H \mid H$ is a closed subgroup of $G$ with $\mathbb{S}(\mathscr{B}) \subset H \subset \mathbb{S}(\mathscr{A})]$. Then the map $\mathscr{F}$ $\rightarrow \mathscr{S}(\mathscr{F})(\mathscr{F} \in F)$ is a bijective map of $F$ onto $G$, its inverse being the map $H \rightarrow \mathfrak{U}(H) \cap \mathscr{B}$.

In order to state the Furstenberg structure theorem I must introduce the notion of an isometric extension. 
3.10 Definition [8]. Let $(X, T)$ and $(Y, T)$ be transformation groups. Then $(X, T)$ is an isometric extension of $(Y, T)$ if there exists a homomorphism $\Pi$ of $(X, T)$ onto $(Y, T)$ (i.e. a continuous surjective map such that $\Pi(x t)=\Pi(x) t$, $(x \in X, t \in T)$ ) and a continuous real valued function $\rho$ defined on the subset $A=\left[\left(x_{1}, x_{2}\right) \mid \Pi x_{1}=\Pi x_{2}\right]$ of $X \times X$ such that $\rho$ restricted to $\Pi^{-1}(y) \times \Pi^{-1}(y)$ is a metric on $\Pi^{-1}(y)$ for all $y \in Y$ and $\rho\left(x_{1}, x_{2}\right)=\rho\left(x_{1} t, x_{2} t\right)$ for all pairs $\left(x_{1}, x_{2}\right)$ in $A$ and all $t$ in $T$.

The isometric extension is nontrivial if $\Pi$ is not one-one.

When $\mathscr{A}$ and $\mathscr{B}$ are $T$-subalgebras of $\mathscr{C}$ with $\mathscr{A} \subset \mathscr{B}$ and I say that $\mathscr{B}$ is an isometric extension of $\mathscr{A}$, the homomorphism $\Pi$ of $(|\mathscr{B}|, T)$ onto $(|\mathscr{A}|, T)$ will always be understood to be the restriction map; i.e. $\Pi(\phi)=\phi \mid \mathscr{A}(\phi \in|\mathscr{B}|)$.

3.11 Proposition [Furstenburg 8]. Let $(X, T)$ be a minimal, distal transformation group with compact metric phase space $X$. Then there exists an ordinal number $v$, minimal, distal, transformation groups $\left(X_{\alpha}, T\right)(\alpha \leqq \nu)$ and surjective homomorphisms $\Pi_{\beta}^{\alpha}: X_{\alpha} \rightarrow X_{\beta}(\beta<\alpha<\nu)$ such that:

(I) $X_{\alpha+1}$ is an isometric extension of $X_{\alpha}$ with respect to the homomorphism $\Pi_{\alpha}^{\alpha+1}(\alpha+1<\nu)$;

(II) $X_{\alpha}$ is the inverse limit of $\left(X_{\beta}, \beta<\alpha\right)$ for every limit ordinal $\alpha<\nu$;

(III) $\left(X_{0}, T\right)$ is the trivial transformation group;

(IV) $X_{v}=X$.

Knapp [12] has observed that one can weaken slightly the condition that $X$ be a metric space. I would now like to restate Proposition 3.11 in terms of $T$-subalgebras of $\mathscr{C}$ incorporating Knapp's generalization. Notice that to say that $X$ is compact metric is equivalent to saying that the algebra of continuous real valued functions on $X$ is separable in the sup topology.

3.12 Definition. Let $\mathscr{A}$ be a $T$-subalgebra of $\mathscr{C}$. Then $\mathscr{A}$ is quasi-separable if the $T$-subalgebra, $\{f\}$, generated by $f$ is separable for all $f \in \mathscr{A}$.

Knapp has also observed that if $T$ can be provided with a $\sigma$-compact topology making the map $(\phi, t) \rightarrow \phi t$ of $|\mathscr{A}| \times T$ into $|\mathscr{A}|$ continuous, then $\mathscr{A}$ is quasiseparable. Thus if $T$ is countable, every $T$-subalgebra of $\mathscr{C}$ is quasi-separable.

Proposition 3.13 is essentially a rephrasing of Proposition 3.11 .

3.13 Proposition. Let $\mathscr{A}$ be a minimal, distal, quasi-separable $T$-subalgebra of $\mathscr{C}$. Then there exists an ordinal $\nu$ and T-subalgebras $\mathscr{A}_{\alpha}(\alpha \leqq \nu)$ such that:

(I) $\mathscr{A}_{\alpha} \subset \mathscr{A}_{\beta}(\alpha \leqq \beta<\nu)$;

(II) $\mathscr{A}_{\alpha+1}$ is an isometric extension of $\mathscr{A}_{\alpha}(\alpha+1 \leqq \nu)$;

(III) $\mathscr{A}_{\alpha}=\operatorname{cl}\left(\bigcup_{\beta<\alpha} \mathscr{A}_{\beta}\right)$ ( $\alpha$ limit ordinal $\left.<\nu\right)$;

(IV) $\mathscr{A}_{0}=R$;

(V) $\mathscr{A}_{v}=\mathscr{A}$.

4. In this section I would like to use the results of $\S 2$ to unify those of $\S 3$ and to generalize Proposition 3.13. 
First I would like to make a few remarks about bitransformation groups $(H, X, T)$. Notice that in Definition 3.6 no mention is made of any topology on $H$ or $T$. For most of the situations encountered in topological dynamics these topologies play a minor role and indeed may be assumed discrete. However, even if $X$ is compact Hausdorff, the orbit space $X / H$ need not be Hausdorff. It is clear that in "practice" it would be nice to know that $X / H$ is indeed Hausdorff. One condition which will guarantee this when $X$ is compact $T_{2}$ is that $H$ can be provided with a compact Hausdorff topology $\mathscr{T}$ such that the map $(h, x) \rightarrow h x$ of $(H, \mathscr{T}) \times X \rightarrow X$ is continuous. If such a $\mathscr{T}$ exists, it must be equal to the topology of pointwise convergence, provided that $H$ acts effectively on $X$. In analogy with fiber space theory it seems natural to call such bitransformation groups principal. (Recall that $H$ acts freely on $X$ if $h x=x$ for some $h \in H$ and some $x \in X$ implies $h$ is the identity of $H$.)

4.1 Definition. Let $(H, X, T)$ be a bitransformation group and $(Y, T)$ a transformation group. Then $(H, X, T)$ is a principal extension of $(Y, T)$ with group $H$ if

(I) $H$ acts freely on $X$,

(II) there exists a compact Hausdorff topology $\mathscr{T}$ on $H$ such that the map $(h, x) \rightarrow h x$ of $(H, \mathscr{T}) \times X$ into $X$ is continuous, and

(III) $(X / H, T)$ is isomorphic to $(Y, T)$.

The bitransformation group $(H, X, T)$ is principal if it is a principal extension of $(X / H, T)$ with group $H$.

4.2 Remarks. 1. Let $(H, X, T)$ be a bitransformation group such that $(X, T)$ is minimal and suppose the action of $H$ on $X$ is effective (i.e. $h x=x$ for all $x \in X$ if and only if $h$ is the identity of $H)$. Then it follows from the minimality of $(X, T)$ and the fact that $[x \mid h x=x]$ is a closed $T$-invariant subset of $X$, that $H$ acts freely on $X$.

2. Let $(H, X, T)$ be principal, $K$ a closed normal subgroup of $H$, then $(H / K, X / K, T)$ is principal.

The purpose of the next two lemmas and propositions is to exhibit the relationship between principal and isometric extensions.

4.3 Standing notation. For the remainder of this section the following notation will be in force: $(H, X, T)$ will denote a principal bitransformation group with compact Hausdorff phase space $X,(Y, T)$ and $\Pi$ the canonical map of $X$ onto $Y$, $(Z, T)$ a transformation group "in between" $(X, T)$ the transformation group $(X / H, T)$ and $(Y, T)$ i.e. I assume the existence of homomorphisms $\phi$ and $\psi$ of $(X, T)$ onto $(Z, T)$ and $(Z, T)$ onto $(Y, T)$ respectively such that the diagram

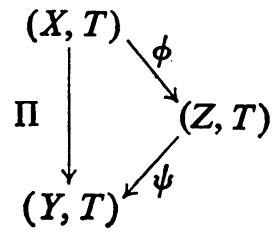


is commutative, finally $y_{0}$ will denote a fixed point of $Y$ and $F$ the subset $\psi^{-1}\left(y_{0}\right)$ of $Z$.

In order to avoid the trivial situation I assume $H \neq\{e\}$, the identity of $H$.

4.4 Proposition. If $H$ is metrizable, then $(X, T)$ is an isometric extension of $(Y, T)$.

Proof. Let $d$ be an invariant metric on $H, S=[(a, b) \mid a, b \in X, a \in H b]$. Then the real valued function $\rho$ on $S$ such that $\rho(a, b)=d(h, e)$ where $(a, b) \in S$ and $h$ is the unique element of $H$ with $a=h b$ satisfies the requirements of definition 3.10.

4.5 COROLlaRY. There exists a nontrivial isometric extension $(W, T)$ of $(Y, T)$ and homomorphisms $f$ and $g$ of $(X, T)$ onto $(W, T)$ and $(W, T)$ onto $(Y, T)$ respectively such that the diagram

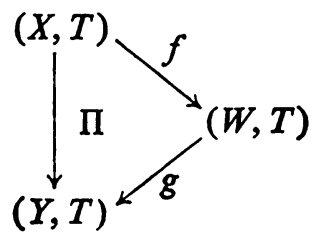

is commutative. Moreover $(W, T)$ may be chosen to be a principal extension of $(Y, T)$, the group of which is a Lie group.

Proof. We can always find a closed normal subgroup $K$ of $H$ such that $L=H / K$ is a Lie group not reduced to the identity. (Of course the identity component of $L$ may be the identity in which case $L$ is finite.)

Set $W=X / K$. Then $Y=W / L$ and $(L, W, T)$ is a principal extension of $(Y, T)$. Also, $(W, T)$ is an isometric extension of $(Y, T)$ by 4.4. (The maps $f$ and $g$ are the canonical ones.)

Now let us assume that $(X, T)$ is minimal. Let $h \in H$. Then the set $[x \mid \phi(h x)$ $=\phi(x)]$ is a closed $T$-invariant subset of $X$. Hence if $\phi\left(h x_{0}\right)=\phi\left(x_{0}\right)$ for some $x_{0} \in X$, then $\phi(h x)=\phi(x)$ for all $x \in X$.

Now let $x_{0} \in X$ with $\Pi\left(x_{0}\right)=y_{0}$, let $z \in F=\psi^{-1}\left(y_{0}\right)$ and $h \in H$. I wish to define an action of $H$ on $F$. There exists $k \in H$ such that $\phi\left(k x_{0}\right)=z$. Set $h z=\phi\left(k h^{-1} x_{0}\right)$.

To see that $h z$ is well defined, let $l \in H$ with $\phi\left(l x_{0}\right)=z$. Then $\Pi\left(l x_{0}\right)=\Pi\left(k x_{0}\right)$ whence there exists $a \in H$ with $k=a l$. Then $\phi\left(a l x_{0}\right)=\phi\left(k x_{0}\right)=\phi\left(l x_{0}\right)$, whence $\phi(a x)=\phi(x)(x \in X)$. Set $x=l h^{-1} x_{0}$. Then $\phi\left(k h^{-1} x_{0}\right)=\phi\left(a l h^{-1} x_{0}\right)=\phi\left(l h^{-1} x_{0}\right)$.

The proof that $(H, F)$ is a transformation group and that the map $(h, z) \rightarrow h z$ of $H \times F$ into $F$ is continuous is straightforward and I shall omit it.

The map $((x, z), t) \rightarrow(x t, z)$ of $X \times F \times T \rightarrow X \times F$ defines an action of $T$ on. $X \times F$ which together with the diagonal action of $H$ on $X \times F$ makes $(H, X \times F, T)$ into a bitransformation group.

I now wish to show that $((X \times F) / H, T)$ is isomorphic to $(Z, T)$. To this end let $z_{0}=\phi\left(x_{0}\right)$. Let $(x, z) \in X \times F$. Then there exists $h \in H$ with $h z_{0}=z$. Set $\phi(x, z)$ $=\phi\left(h^{-1} x\right)$. Now suppose $k \in H$ with $k z_{0}=z$. Then $z=\phi\left(k^{-1} x_{0}\right)=\phi\left(h^{-1} x_{0}\right)=$ $\phi\left(h^{-1} k k^{-1} x_{0}\right)$ whence $\phi(u)=\phi\left(h^{-1} k u\right)(u \in X)$. 
Set $u=k^{-1} x$. Then $\phi\left(k^{-1} x\right)=\phi\left(h^{-1} k k^{-1} x\right)=\phi\left(h^{-1} x\right)$, whence $\phi$ is well defined. Again I shall leave the proof of the continuity of $\phi$ to the reader.

Clearly $\phi$ is onto. Now let $l \in H$ and $(x, z) \in X \times F$. Let $h \in H$ with $h z_{0}=z$. Then $l h z_{0}=l z$ whence $\phi(l(x, z))=\phi(l x, l z)=\phi\left((l h)^{-1} l x\right)=\phi\left(h^{-1} x\right)=\phi(x, z)$. Thus $\phi$ induces a homomorphism $\hat{\phi}$ of $((X \times F) / H, T)$ onto $(Z, T)$.

Finally suppose $\phi(x, z)=\phi\left(x_{2}, z_{2}\right)$ for two points $\left(x_{1}, z_{1}\right)$ and $\left(x_{2}, z_{2}\right)$ of $X \times F$. Let $z_{1}=h_{1} z_{0}$ and $z_{2}=h_{2} z_{0}$. Then $\phi\left(h_{1}^{-1} x_{1}\right)=\phi\left(h_{2}^{-1} x_{2}\right)$. This implies that $\Pi\left(x_{1}\right)$ $=\Pi\left(h_{1}^{-1} x_{1}\right)=\Pi\left(h_{2}^{-1} x_{2}\right)=\Pi\left(x_{2}\right)$. Hence there exists $l \in H$ with $x_{2}=l x_{1}$. Then $\phi\left(h_{1}^{-1} x_{1}\right)=\phi\left(h_{2}^{-1} l x_{1}\right)$ whence $\phi\left(h_{1}^{-1} x\right)=\phi\left(h_{2}^{-1} l x\right)$ for all $x \in X$. Now set $x=l^{-1} x_{0}$. Then $\phi\left(h_{1}^{-1} l^{-1} x_{0}\right)=\phi\left(h_{2}^{-1} x_{0}\right)$, which is another way of writing that $l z_{1}=z_{2}$. Thus $l\left(x_{1}, z_{1}\right)=\left(l x_{1}, l z_{1}\right)=\left(x_{2}, z_{2}\right)$. This completes the proof of the following proposition. (The proof that the various diagrams involved are commutative is straightforward.)

4.6 Proposition. If $(X, T)$ is minimal then there exists an action of $H$ on $F$ and an isomorphism $\hat{\phi}$ of $((X \times F) / H, T)$ onto $(Z, T)$ such that the diagram

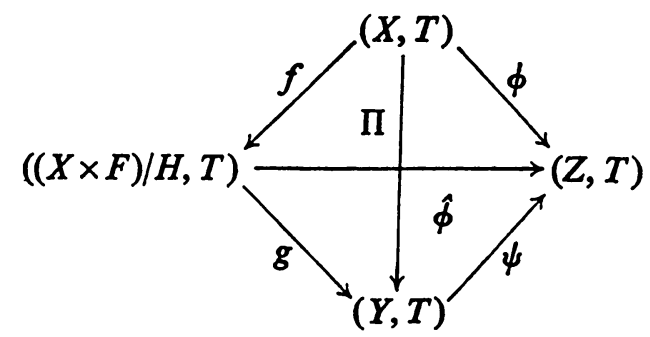

is commutative; where $f(x)=\eta\left(x, z_{0}\right)(x \in X)$ and $g \eta(x, z)=\Pi(x)(x \in X, z \in F)$ and $\eta$ is the canonical map of $X \times F$ onto $(X \times F) / H$.

4.7 Proposition. Let $(X, T)$ be minimal and $F$ metrizable. Then $(Z, T)$ is an isometric extension of $(Y, T)$.

Proof. By Proposition 4.6 we may assume that $(Z, T)=((X \times F) / H, T)$. Let $\sigma$ be a metric on $F$ and set $d(u, v)=\sup [\sigma(h u, h v) \mid h \in H]$. Then $d$ is an $H$-invariant metric on $F$.

Using the notation of Proposition 4.6 set $S=[(a, b) \mid a, b \in(X \times F) / H, g(a)=g(b)]$. I wish to define a real valued function $\rho$ on $S$ which satisfies the conditions of Definition 3.10 .

To this end let $(a, b) \in S$. Choose elements $\left(x_{1}, z_{1}\right),\left(x_{2}, z_{2}\right)$ in $X \times F$ such that $\eta\left(x_{1}, z_{1}\right)=a$ and $\eta\left(x_{2}, z_{2}\right)=b$. Since $g(a)=g(b)$, there exists a unique $l \in H$ with $x_{2}=l x_{1}$. Then set $\rho(a, b)=d\left(l z_{1}, z_{2}\right)$. To see that $\rho$ is well defined suppose $\eta\left(x_{1}^{\prime}, z_{1}^{\prime}\right)$ $=a, \eta\left(x_{2}^{\prime}, z_{2}^{\prime}\right)=b$ and $x_{2}^{\prime}=l^{\prime} x_{1}^{\prime}$. We must show that $d\left(l^{\prime} z_{1}^{\prime}, z_{2}^{\prime}\right)=d\left(l z_{1}, z_{2}\right)$.

Since $\eta\left(x_{i}, z_{i}\right)=\eta\left(x_{i}^{\prime}, z_{i}^{\prime}\right)(i=1,2)$, there exist $h_{1}, h_{2} \in H$ with $\left(x_{i}^{\prime}, z_{i}^{\prime}\right)=h_{i}\left(x_{i}, z_{i}\right)$ $(i=1,2)$. Then $x_{2}^{\prime}=h_{2} x_{2}=h_{2} l x_{1}=h_{2} l h_{1}^{-1} x_{1}^{\prime}$ shows that $l^{\prime}=h_{2} l h_{1}^{-1}$. Thus $d\left(l^{\prime} z^{\prime}, z_{2}^{\prime}\right)$ $=d\left(h_{2} l h_{1}^{-1} z_{1}^{\prime}, z_{2}^{\prime}\right)=d\left(h_{2} l h_{1}^{-1} h_{1} z_{1}, h_{2} z_{2}\right)=d\left(h_{2} l z_{1}, h_{2} z_{2}\right)=d\left(l z_{1}, z_{2}\right)$ by the $H$ invariance of $d$.

The proof that the above defined $\rho$ does indeed satisfy 3.10 is tedious but straightforward and will be omitted. 
4.8 Proposition. Let $(X, T)$ be minimal and let $(Z, T)$ be a nontrivial extension of $(Y, T)$ (i.e. $\left.F \neq\left\{z_{0}\right\}\right)$. Then there exist a transformation group $(W, T)$ and homomorphisms $\alpha, \beta$ of $(Z, T)$ onto $(W, T)$ and $(W, T)$ onto $(Y, T)$ such that $\psi=\beta \circ \alpha$ and $(W, T)$ is a nontrivial isometric extension of $(Y, T)$.

Proof. Again we may assume that $Z=(X \times F) / H$ and $\psi=g$. Since $F \neq\left\{z_{0}\right\}$ there exists a neighborhood $V$ of the identity of $H$ such that $V z_{0} \neq F$. There exists a closed normal subgroup $K$ of $H$ such that $K \subset V$ and $H / K=L$ is metrizable.

Set $N=F / K$. Then since $H$ acts transitively on $F, L$ acts transitively on $L$. Hence $N$ is metrizable. Of course $H$ also acts on $N$ and we may form $W=(X \times N) / H$. Then the canonical map of $F$ onto $N$ induces a map $\alpha$ of $(X \times F) / H$ onto $(X \times N) / H$. Set $\beta(\rho(x, z))=\Pi(x)$ for all $x \in X, z \in N$, where $\rho$ is the canonical map of $X \times N$ onto $W$. Then $g=\beta \circ \alpha$ and $(W, T)$ is an isometric extension of $(Y, T)$ by 4.7 . It is nontrivial because $K z_{0} \neq F$.

4.9 I would now like to prove the main result of this paper. Again in order to avoid needless repetition I shall introduce some notation which will be in force till the end of this section.

Thus $\mathscr{F}$ will denote a $T$-subalgebra of $\mathscr{M}, K$ a $\tau(\mathscr{C})$-closed subgroup of $G$ with $\mathscr{F} K \subset \mathscr{F}, \mathscr{L}$ the subset $[f \mid f \in \mathscr{F}$; the maps $\alpha \rightarrow\langle f, \alpha p\rangle$ of $(K, \tau(\mathscr{F}))$ into $R$ are continuous for all $p \in \beta T], \mathscr{K}=[f \mid f \in \mathscr{F}, f \alpha=f,(\alpha \in K)]$. The main result then states that $\mathscr{L}$ is a principal extension of $\mathscr{K}$ with group $K / K \cap \mathbb{S}(\mathscr{L})$.

The proof of the following lemma is straightforward.

4.10 LemMa. $\mathscr{L}$ is a T-subalgebra of $\mathscr{F}$ such that $\mathscr{K} \subset \mathscr{L}$ and $\mathscr{L} K \subset \mathscr{L}$.

4.11 LEMMA. $K \cap \mathbb{S}(\mathscr{L})$ is a $\tau(\mathscr{F})$-closed, normal subgroup of $K$ and

$$
(K /(K \cap \mathbb{S}(\mathscr{L})), \tau(\mathscr{F}))
$$

is a compact Hausdorff topological group.

Proof. Set $L=\mathscr{S}(\mathscr{L})$. Let $\alpha \in \operatorname{cls} \mathscr{F} L, f \in \mathscr{L}$. Since $\mathscr{L} \subset \mathscr{F}, f_{L} \omega \leqq f \alpha \leqq f^{L} \omega$. But $f_{L}=f=f^{L}$. Hence $f=f \omega=f \alpha$; i.e. $\alpha \in \mathbb{S}(\mathscr{L})=L$. Hence $K \cap \mathbb{S}(\mathscr{L})$ is a $\tau(\mathscr{F})$-closed subgroup of $K$.

Let $\alpha \in K, \beta \in K \cap \mathbb{S}(\mathscr{L}), f \in \mathscr{L}$. Then $f \alpha \in \mathscr{L}$ implies that $f \alpha \beta \alpha^{-1}=f \alpha \alpha^{-1}$ $=f \omega=f$. Thus $\alpha \beta \alpha^{-1} \in K \cap \mathbb{S}(\mathscr{L})$ whence $K \cap \mathbb{S}(\mathscr{L})$ is a normal subgroup of $K$.

By 2.12 and 2.10 it suffices to show that $Q=Q(K, \mathscr{F})$ is contained in $L$. Let $f \in \mathscr{L}, p \in \beta T, \varepsilon>0$ and $I$ the closed interval about $\langle f, p\rangle$ of length $\varepsilon$. Since $\langle f, p\rangle$ $=\langle f, \omega p\rangle$ and the map $\alpha \rightarrow\langle f, \alpha p\rangle$ of $(K, \mathscr{F})$ into $R$ is continuous there exists a $\tau(\mathscr{F})$ neighborhood $V$ of $\omega$ such that $\langle f, \beta p\rangle \in I$ for all $\beta \in \operatorname{cls} \mathscr{F} V$. Hence $\langle f, \beta p\rangle \in I$, $(\beta \in Q)$. Since $\varepsilon$ and $p$ were arbitrary, this implies that $f=f \beta(\beta \in Q, f \in \mathscr{L})$. Thus $Q \subset \mathbb{E}(\mathscr{L})=L$. The proof is completed.

4.12 Proposition. Let $L=K \cap \mathscr{S}(\mathscr{L}), S=K / L, \chi: K \rightarrow S$ the canonical map. Then the map $\eta: S \times|\mathscr{L}| \rightarrow|\mathscr{L}|$ such that $\eta(\chi(\alpha), x)=\alpha x(\alpha \in K, x \in|\mathscr{L}|)$ makes $(S,|\mathscr{L}|, T)$ into a principal bitransformation group. 
Note: since $\mathscr{L} K \subset \mathscr{L}$ then the map $f \rightarrow f \alpha x$ which I denote $\alpha x$ is a well-defined element of $|\mathscr{L}|$ for all $\alpha \in K$ and $x \in|\mathscr{L}|$. This defines a map $\zeta: K \times|\mathscr{L}| \rightarrow|\mathscr{L}|$ namely $\zeta(\alpha, x)=\alpha x(\alpha \in K, x \in|\mathscr{L}|)$. Then the following diagram defines $\eta$

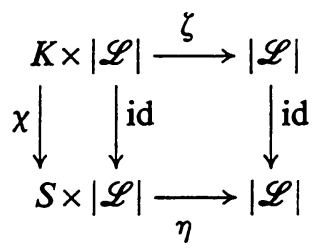

Proof. Let $\alpha, \beta \in K$ with $\chi(\alpha)=\chi(\beta)$ and let $x \in|\mathscr{L}|$. Then $\alpha \beta^{-1} \in L$ whence $f \alpha=f \beta(f \in \mathscr{L})$. Thus $f \alpha x=f \beta x(f \in \mathscr{L})$ whence $\alpha x=\beta x$. Hence $\eta$ is well defined.

Recall that the topology on $|\mathscr{L}|$ is the smallest one making the functions $x \rightarrow\langle f x, e\rangle$ continuous for all $f \in \mathscr{L}$. Since $\mathscr{L}_{\alpha} \subset \mathscr{L}(\alpha \in K)$, this implies that the maps $x \rightarrow \eta(s, x)(x \in|\mathscr{L}|)$ of $|\mathscr{L}|$ into $|\mathscr{L}|$ are continuous for all $s \in S$.

Moreover, by the definition of $\mathscr{L}$ and the topology on $|\mathscr{L}|$, the maps $\alpha \rightarrow \alpha x$ of $(K, \tau(\mathscr{F}))$ into $|\mathscr{L}|$ are continuous for all $x \in|\mathscr{L}|$. Consequently the maps $s \rightarrow \eta(s, x)$ of $(S, \tau(\mathscr{F}))$ into $|\mathscr{L}|$ are continuous $(x \in|\mathscr{L}|)$. Hence $\eta$ is continuous [2].

Since $(s x) t=s(x t)(s \in S, x \in|\mathscr{L}|, t \in T)$, it remains only to be shown that $S$ acts freely on $|\mathscr{L}|$. Now $\mathscr{L} \subset \mathscr{M}$ implies that $(|\mathscr{L}|, T)$ is minimal. Hence we need only show that $S$ acts effectively on $|\mathscr{L}|$. To this end let $s \in S$ be such that $s x=x(x \in|\mathscr{L}|)$. Let $\alpha \in K$ with $\chi(\alpha)=s$ and let $x_{0}$ be the inclusion mapping of $\mathscr{L}$ into $\mathscr{C}$. Then $x_{0} \in|\mathscr{L}|$ and $s x_{0}=x_{0}$ implies that $f \alpha=f(f \in \mathscr{L})$. Hence $\alpha \in L$ and $s$ is the identity element of $S$. The proof is completed.

4.13 LemMA. Let $x, y \in|\mathscr{L}|$ with $x|\mathscr{K}=y| \mathscr{K}$. Then there exists $\alpha \in K$ with $x=\alpha y$.

Proof. Let $S$ be as in 4.12. Assume that $x \notin S y(S y=\eta(S, y))$. Then the two closed subsets $S x$ and $S y$ are disjoint. Hence there exists $f \in \mathscr{L}$ such that $\langle f \alpha x, e\rangle=0$ and $\langle f \alpha y, e\rangle=1(\alpha \in K)$.

Since $S$ acts on $|\mathscr{L}|$, it acts on $\mathscr{L}$. (The action of $S$ on $\mathscr{L}$ is given by $f \rightarrow f s$ $(f \in \mathscr{L}, s \in S)$ where $f s=f \alpha$ for some $\alpha \in K$ with $\chi(s)=\alpha$.) Proposition 4.12 implies that the map $s \rightarrow f s$ of $S$ into $\mathscr{L}$ is continuous when $\mathscr{L}$ is provided with the supremum norm. Since the image of $S$ under the above map is compact, there exists $g \in \mathscr{L}$ such that (I) $\lambda(g)=\int_{s} \lambda(f s) d \mu(s)[1, \S 4$, Proposition 2] for all continuous linear functionals $\lambda$ on $\mathscr{L}$ where $\mu$ is Haar measure on $S$.

Let $\alpha \in K, t \in T$. Then the map $h \rightarrow\langle h, \alpha t\rangle(h \in \mathscr{L})$ of $\mathscr{L}$ into $R$ is continuous and linear. Hence (I) gives that $\langle g, \alpha t\rangle=\int_{S}\langle f s, \alpha t\rangle d \mu(s)$. Let $\chi(\alpha)=r \in S$. Then $\langle f s, \alpha t\rangle=\langle f s \alpha, t\rangle=\langle f s r, t\rangle$ and $\int_{S}\langle f s r, t\rangle d \mu(s)=\int_{S}\langle f s, t\rangle d \mu(s)=\langle g, t\rangle$ by the invariance of $\mu$. Thus $g \alpha=g(\alpha \in K)$ whence $g \in \mathscr{K}$.

Again, applying (I) to the linear functionals $h \rightarrow\langle h x, e\rangle$ and $h \rightarrow\langle h y, e\rangle$ we see that $\langle g x, e\rangle=\int_{S}\langle f s x, e\rangle d \mu(s)=0$ and $\langle g y, e\rangle=\int_{S}\langle f s y, e\rangle d \mu(s)=1$. But this contradicts the facts that $g \in \mathscr{K}$ and $x|\mathscr{K}=y| \mathscr{K}$. 
4.14 Proposition. Let $\mathscr{F}$ be a $T$-subalgebra of $\mathscr{M}$ and $K$ a $\tau(\mathscr{C})$-closed subgroup of $G$ with $\mathscr{F} K \subset \mathscr{F}$. Then $\mathscr{L}$ is a principal extension of $\mathscr{K}$ with group $K /(K \cap \mathbb{S}(\mathscr{L}))$ where $\mathscr{L}=[f \mid f \in \mathscr{F}$ and the maps $\alpha \rightarrow\langle f, \alpha p\rangle$ of $(K, \tau(\mathscr{F}))$ into $R$ are continuous for all $p \in \beta T]$ and $\mathscr{K}=\mathscr{F} \cap \mathfrak{U}(K)$.

Proof. By 4.13 all we need show is that the restriction mapping $\eta$ of $|\mathscr{L}|$ onto $|\mathscr{K}|$ induces an isomorphism of $(|\mathscr{L}| / S, T)$ onto $(|\mathscr{K}|, T)$.

Let $x \in|\mathscr{L}|$ and $s \in S$. Then $\eta(s x)=\alpha x \mid \mathscr{K}$ where $\alpha \in K$ with $\chi(\alpha)=s$. But $f \alpha=f$ $(f \in \mathscr{K})$ by the definition of $\mathscr{K}$. Hence $\eta$ induces a homomorphism $\bar{\eta}$ of $(|\mathscr{L}| / S, T)$ onto $(|\mathscr{L}|, T)$.

Now suppose $\eta(x)=\eta(y)$ for two elements $x, y$ of $|\mathscr{L}|$. Then $x|\mathscr{K}=y| \mathscr{K}$ whence $x=\alpha y$ for some $\alpha \in K$ by 4.13 . Thus $x$ and $y$ determine the same element of $|\mathscr{L}| / S$. Hence $\bar{\eta}$ is injective. The proof is completed.

In order to apply to 4.14 one must be able to compute $\mathscr{L}$. Moreover 4.14 says something nontrivial only when $\mathscr{L} \neq \mathscr{K}$. Thus one must be able to compute $\mathscr{L}$ and prove that it is not $\mathscr{K}$. This is what I would like to do in the case of group-like extensions.

Thus let $\mathscr{A}, \mathscr{B}$ be $T$-subalgebras of $\mathscr{M}$ with $\mathscr{A} \subset \mathscr{B}$ and $\mathscr{B}$ a group-like extension of $\mathscr{A}$. The natural candidates for $\mathscr{F}$ and $K$ of the preceding discussion are $\mathscr{B}$ and $\mathscr{S}(\mathscr{A})$ respectively. However, in general $\mathscr{B} \mathscr{S}(\mathscr{A})$ is not contained in $\mathscr{B}$. (This is true only if $\mathscr{B}$ is a group-extension of $\mathscr{A}$.) Thus the first step in the analysis is to enlarge $\mathscr{B}$ to a group extension, $\mathscr{S}$ of $\mathscr{A}$.

4.15 LEMMA. Let $\mathscr{S}$ be the $T$-subalgebra of $\mathscr{M}$ generated by $\cup[\mathscr{B} \alpha \mid \alpha \in \mathbb{S}(\mathscr{A})]$. Then $\mathscr{S}$ is a group extension of $\mathscr{A}$.

Proof. It is clear from the definition of $\mathscr{S}$ that $\mathscr{S} \mathscr{S}(\mathscr{A}) \subset \mathscr{S}$. Thus it suffices to show that $\mathscr{S}$ is a group-like extension of $\mathscr{S}$.

To this end let $p \in \beta T, w \in M$ with $w^{2}=w$ be such that $p|\mathscr{A}=p w| \mathscr{A}$. Set $\mathscr{G}$ $=[f \mid f \in \mathscr{S}, f p=f p w]$. Then it is immediate that $\mathscr{S}$ is a $T$-subalgebra of $\mathscr{S}$.

Since $\mathscr{B}$ is a group-like extension of $\mathscr{A}, p|\mathscr{B}=p w| \mathscr{B}$. Hence $\mathscr{B} \subset \mathscr{G}$. Now let $\alpha \in \mathscr{S}(\mathscr{A})$. Then $\alpha p|\mathscr{A}=p| \mathscr{A}=p w|\mathscr{A}=\alpha p w| \mathscr{A}$. Hence $\alpha p|\mathscr{B}=\alpha p w| \mathscr{B}$, whence $\mathscr{B} \alpha \subset \mathscr{G}$. Thus $\mathscr{B}_{\alpha} \subset \mathscr{G}(\alpha \in \mathbb{S}(\mathscr{A}))$, whence $\mathscr{G}=\mathscr{S}$. The proof is completed.

4.16 LemMa. Let $\mathscr{A}, \mathscr{B}$, and $\mathscr{S}$ be as above. Then

$$
\mathscr{A}=[f \mid f \in \mathscr{S}, f \alpha=f(\alpha \in \mathscr{S}(\mathscr{A}))] \text {. }
$$

Proof. Set $\mathscr{G}=[f \mid f \in \mathscr{S}, f \alpha=f(\alpha \in \mathscr{S}(\mathscr{A}))]$. Then $\mathscr{G}$ is a $T$-subalgebra of $\mathscr{S}$ such that $\mathscr{A} \subset \mathscr{G}$. This implies that $\mathbb{S}(\mathscr{G}) \subset \mathscr{S}(\mathscr{A})$. But it is evident from the definition of $\mathscr{G}$ that $\mathscr{S}(\mathscr{A}) \subset \mathscr{S}(\mathscr{G})$. Hence $\mathscr{S}(\mathscr{A})=\mathscr{S}(\mathscr{G})$. Since $\mathscr{S}$ is a group-like extension of $\mathscr{A}$ and $\mathscr{A} \subset \mathscr{G} \subset \mathscr{S}, \mathscr{G}$ is also a group-like extension of $\mathscr{A}$ [7, Proposition 9]. Hence $\mathscr{G}=\mathscr{A}$. The proof is completed.

Lemmas 4.15 and 4.16 indicate that we may apply the general theory with $\mathscr{F}=\mathscr{S}, K=\mathscr{S}(\mathscr{A})$ and that if we do this $\mathscr{K}$ turns out to be $\mathscr{A}$. 
Now I would like to determine the $T$-subalgebra $\mathscr{W}=[f \mid f \in \mathscr{S}$ and the maps $\alpha \rightarrow\langle f, \alpha p\rangle$ of $(\mathscr{S}(\mathscr{A}), \tau(\mathscr{S})$ ) into $R$ are continuous for all $p \in \beta T$ ] which plays the role of $\mathscr{L}$.

Set $A=\mathscr{S}(\mathscr{A}), Q=Q(A, \mathscr{S}), \mathscr{Q}=[f \mid f \in \mathscr{S}, f \alpha=f(\alpha \in Q)]$. Then I shall show that $\mathscr{W}=\mathscr{Q}$.

The proof of Lemma 4.12 shows (in the present notation) that $Q \subset \&(W)$. Hence $\mathscr{W} \subset \mathcal{Q}$.

Now suppose $f \in \mathscr{Q}$. Let $p \in \beta T$ and $\left\{\alpha_{n}\right\}$ a net of elements of $A$ such that $\alpha_{n}$ $\rightarrow \alpha \in A$ in the topology $\tau(\mathscr{S})$. I wish to show that $\left\langle f, \alpha_{n} p\right\rangle \rightarrow\langle f, \alpha p\rangle$.

First observe that since $\beta \omega=\beta(\beta \in G),\langle f, \beta p\rangle=\langle f, \beta \omega p\rangle(\beta \in G)$. Thus we may replace $p$ by $\omega p$ in the above paragraph. This implies that we may suppose that $p=\omega p$, i.e. $p \in M$.

Since $M$ is compact, we may suppose that $\alpha_{n} p$ converges to $q \in M$. Then of course $\left(^{*}\right)\left\langle h, \alpha_{n} p t\right\rangle \rightarrow\langle h, q t\rangle$ for all $h \in \mathscr{C}, t \in T$. If we take $h$ in $\mathscr{A}$, this shows that $h p=h q$ since $\alpha_{n} \in \mathbb{S}(\mathscr{A})$. Thus $p|\mathscr{A}=q| \mathscr{A}$. By [7, Lemma 23] there exists $\beta \in A$ with $\beta p=q$.

I shall now show that $\alpha^{-1} \beta \in Q$. To this end let $\gamma \in G$ with $p \gamma=\omega$ (such exists since $p \in M$ and $p M=M$, see [4] for details). I claim that there is a subnet of $\left\{\alpha_{n} p \gamma\right\}$ $=\left\{\alpha_{n}\right\}$ which converges to $q \gamma$ in the topology $\tau(\mathscr{S})$. Indeed, if this were not so there would exist an index $k$ such that $q \gamma \notin \operatorname{cls} \mathscr{S} N$, where $N=\left[\alpha_{m} / m \geqq k\right]$.

This leads to a contradiction as follows: Let $h \in \mathscr{S}$. Then $h q \leqq h^{N p}$ since the net $\left\{\alpha_{n} p\right\}$ converges pointwise to $q$ (see Remarks $2.3,1$ ). Then $h q \gamma \leqq h^{N p} \gamma \leqq h^{N} p \gamma=h^{N} \omega$ (by Lemma 1.2, 4). This means that $q \gamma \in N^{h}(h \in \mathscr{S})$, i.e. $q \gamma \in \operatorname{cls} \mathscr{S} N$. Thus I may suppose that $\left\{\alpha_{m}\right\}$ converges to $q \gamma$ in the topology $\tau(\mathscr{S})$. Since $\left\{\alpha_{m}\right\}$ also converges to $\alpha$ in this topology, $\alpha^{-1} q \gamma \in Q$. Now $q \gamma=\beta p \gamma=\beta \omega=\beta$.

Finally, relation $\left(^{*}\right)$ with $h=f$ and $t=e$ shows that $\left\langle f, \alpha_{n} p\right\rangle \rightarrow\langle f, \beta p\rangle=\langle f, \alpha p\rangle$, since $f \in \mathscr{Q}$ implies that $f \alpha=f \beta$ if $\alpha^{-1} \beta \in Q$.

Thus we have proved that $\mathscr{Q}=\mathscr{W}$. Now $\mathscr{S}$ is a group-like extension of $\mathscr{A}, Q$ a closed subgroup of $G$ with $\mathscr{S}(\mathscr{S}) \subset Q \subset \mathscr{S}(\mathscr{A})$ and $\mathscr{Q}=\mathfrak{2}(Q) \cap \mathscr{S}$. Hence by 3.9 (S) $(\mathscr{Q})=Q$.

The above results are collected in the following proposition.

4.17 Proposition. Let $\mathscr{A}, \mathscr{B}$ be $T$-subalgebras of $\mathscr{M}$ with $\mathscr{A} \subset \mathscr{B}$ and $\mathscr{B}$ a grouplike extension of $\mathscr{A}, \mathscr{S}$ the T-subalgebra of $\mathscr{M}$ generated by $\cup[\mathscr{B} \alpha \mid \alpha \in \mathscr{S}(\mathscr{A})]$, $Q=Q(\mathscr{S}(\mathscr{A}), \mathscr{S}), \mathscr{Q}=[f \mid f \in \mathscr{S}, f \alpha=f,(\alpha \in Q)]$. Then $\mathscr{Q}$ is a principal extension of $\mathscr{A}$ with group $\mathscr{E}(\mathscr{A}) / Q$.

The problem now is to show that $Q \neq \mathscr{S}(\mathscr{A})$, for then $\mathscr{Q} \neq \mathscr{A}$ and so $\mathscr{Q}$ is a nontrivial extension of $\mathscr{A}$. It is at this point that the assumption that $\mathscr{B}$ is quasiseparable must be introduced. I do not know whether it is true that $Q \neq \mathscr{S}(\mathscr{A})$ without this assumption.

My present aim is to show that when $\mathscr{B}$ is quasi-separable $\mathscr{S}(\mathscr{B}) Q \neq \mathscr{S}(\mathscr{A})$.

For the remainder of this section I shall retain the notation of Proposition 4.17. 
4.18 Lemma. Let $L$ be a finite subset of $\mathscr{S}$. Set

$$
\langle L, \alpha\rangle=\inf \left[\sum_{f \in L}|\langle f, \alpha t\rangle-\langle f, t\rangle| \mid t \in T\right], \quad(\alpha \in \mathbb{S}(\mathscr{A})) .
$$

Then $L$ is an upper semicontinuous function on $(\circlearrowleft(\mathscr{A}), \tau(\mathscr{S})$ ). (Note that I use the same letter to denote the set and the function associated with the set. This should, however, cause no confusion.)

Proof. Let $a \in R, N=[\alpha \mid \alpha \in \mathbb{S}(\mathscr{A}),\langle L, \alpha\rangle \geqq a]$. I wish to show that $N$ is $\tau(\mathscr{S})$ closed.

Let $\beta \in \operatorname{cls}_{\mathscr{S}} N, t \in T$. Set $\phi=\sum_{f \in L}|t f-\langle f, t\rangle|$; i.e. $\langle\phi, p\rangle=\sum_{f \in L}|\langle f, p t\rangle-\langle f, t\rangle|$ $(p \in \beta T)$. Then $\phi \in \mathscr{S}$, whence (I) $\phi_{N} \omega \leqq \phi \beta$.

Let $\alpha \in N, s \in T$. Then

$$
\begin{aligned}
\langle\phi, \alpha s\rangle & =\sum_{f \in L}|\langle f, \alpha s t\rangle-\langle f, t\rangle| \geqq \sum_{f \in L} \mid\left\langle f, \alpha s t-\langle f, s t\rangle\left|-\sum_{f \in L}\right|\langle f, s t\rangle-\langle f, t\rangle\right| \\
& \geqq a-\sum_{f \in L}|\langle f, s t\rangle-\langle f, t\rangle|
\end{aligned}
$$

since $\alpha \in N$. Thus $\phi \alpha \geqq a-\phi(\alpha \in N)$. Consequently $\phi_{N} \geqq a-\phi$. Combining this with (I) we get $(a-\phi) \omega \leqq \phi \beta$. Evaluating this at $e$ yields $\langle\phi, \beta\rangle \geqq a$ since $\langle\phi, e\rangle=0$. Hence $\sum_{f \in L}|\langle f, \beta t\rangle-\langle f, t\rangle| \geqq a$. Since $t$ was arbitrary this implies that $\langle L, \beta\rangle \geqq a$, whence $\beta \in N$. The proof is completed.

The proof of Corollary 4.19 follows in the classical manner from 4.18.

4.19 COROLLARY. With the same notation as in 4.18 , the set of points of continuity of $L$ is a residual subset of $\mathscr{B}(\mathscr{A})$.

4.20 LEMMA. Let $L$ be a finite subset of $\mathscr{B}$,

$$
\langle L, \alpha\rangle=\inf \left[\sum_{f \in L}|\langle f, \alpha t\rangle-\langle f, t\rangle| \mid t \in T\right] \quad(\alpha \in \mathbb{S}(\mathscr{A})),
$$

$\beta \in \mathbb{S}(\mathscr{B}) Q$. Then $\langle L, \beta\rangle=0$ if $L$ is continuous at $\beta$.

Proof. First observe that $\langle L, \omega\rangle=0$ and that since $L \geqq 0, L$ is continuous at $\omega$ by 4.18 .

Now suppose $L$ is continuous at $\beta=\gamma \delta$ where $\gamma \in \mathbb{S}(\mathscr{B})$ and $\delta \in Q$. Let $\varepsilon>0$. Choose neighborhoods $V$ of $\beta, W$ of $\delta$ and $N$ of $\omega$ such that (I) $\gamma W \subset V$, (II) $|\langle L, \alpha\rangle-\langle L, \beta\rangle|<\varepsilon(\alpha \in V)$, (III) $\langle L, \alpha\rangle\langle\varepsilon(\alpha \in N)$.

Since $\delta \in Q \subset \operatorname{cls}_{\mathscr{S}} N$, there exists $\eta \in W \cap N$. By (I) and (II) $|\langle L, \gamma \eta\rangle-\langle L, \beta\rangle|<\varepsilon$ and by (III) $\langle L, \eta\rangle\langle\varepsilon$. But $L \gamma=L$ since $L \subset \mathscr{B}$ and $\gamma \in \mathscr{S}(\mathscr{B})$. Thus $\langle L, \gamma \eta\rangle=\langle L, \eta\rangle$ whence $\langle L, \beta\rangle\langle 2 \varepsilon$. The proof is completed.

4.21 Lemma. Let $f \in \mathscr{B},\{f\}$ the $T$-subalgebra of $\mathscr{B}$ generated by $f, \mathscr{H}$ a dense subset of $\{f\}, \alpha \in \mathbb{B}(\mathscr{B}) Q$ such that $L$ is continuous at $\alpha$ for all finite subsets $L$ of $\mathscr{H}$. Then $f \alpha=f$. 
Proof. By $4.20\langle L, \alpha\rangle=0$ for all finite subsets $L$ of $\mathscr{H}$. This implies that the set $A_{L}=[x \mid x \in M,\langle h, \alpha x\rangle=\langle h, x\rangle(h \in L)]$ is nonvacuous for all such $L$.

Clearly $A_{L}$ is a closed subset of $M$ and $A_{L} \subset A_{N}$ if $N \subset L$. Hence the family $\left(A_{L} \mid L\right.$ finite subset of $\mathscr{H})$ has the finite intersection property. Consequently there exists $x \in M$ with $\langle h \alpha, x\rangle=\langle h, x\rangle(h \in \mathscr{H})$. Since $\mathscr{H}$ is dense in $\{f\},\langle h \alpha, x\rangle=\langle h, x\rangle$ $(h \in\{f\})$. This implies that $\langle t f \alpha, x\rangle=\langle t f, x\rangle(t \in T)$; i.e. $\langle f \alpha, x t\rangle=\langle f, x t\rangle(t \in T)$. Since $\operatorname{cl}(x T)=M,\langle f \alpha, y\rangle=\langle f, y\rangle(y \in M)$. Thus $\langle f \alpha, t\rangle=\langle f \alpha, \omega t\rangle=\langle f, \omega t\rangle$ $=\langle f \omega, t\rangle=\langle f, t\rangle(t \in T)$. The proof is completed.

4.22 LEMMA. Let $\mathscr{V}$ be the smallest topology on $\mathscr{S}(\mathscr{A})$ making the real valued functions $\alpha \rightarrow\langle f, \alpha\rangle(\alpha \in \mathbb{S}(\mathscr{A}))$ continuous for all $f \in \mathscr{S}$. Then $(1)(\mathscr{S}(\mathscr{A}), \mathscr{V})$ is compact. (2) The maps $\alpha \rightarrow \beta \alpha(\alpha \in \mathbb{S}(\mathscr{A}))$ of $(\mathbb{S}(\mathscr{A}), \mathscr{V})$ into $(\mathscr{S}(\mathscr{A}), \mathscr{V})$ are continuous for all $\beta \in \mathbb{S}(\mathscr{A})$. (3) $\mathscr{V} \supset \tau(\mathscr{S}),(4)(\mathscr{S}(\mathscr{A}) / \mathscr{S}(\mathscr{S}), \mathscr{V})(\mathscr{V}$ here denotes the quotient topology induced by $\mathscr{V}$ ) is compact Hausdorff.

Proof. 1. Let $\mathscr{U}$ be an ultra filter on $\mathscr{S}(\mathscr{A})$. Since $M$ is compact there exists $p \in M$ such that $\mathscr{U} \rightarrow p$ pointwise. Then $f(\mathscr{U}) \rightarrow\langle f, p\rangle(f \in \mathscr{S})$.

Since $\mathscr{U} \subset \mathscr{S}(\mathscr{A}), p \mid \mathscr{A}$ is the identity. Hence there exists [7, Lemma 3] $\alpha \in \mathscr{S}(\mathscr{A})$ with $p|\mathscr{S}=\alpha| \mathscr{S}$. Thus $f(\mathscr{U}) \rightarrow\langle f, \alpha\rangle(f \in \mathscr{S})$.

2. Let $\left(\alpha_{n}\right)$ be a net in $\mathscr{S}(\mathscr{A})$ such that $\alpha_{n} \rightarrow \alpha \in \mathbb{S}(\mathscr{A})$ with respect to $\mathscr{V}$; i.e. $\left\langle f, \alpha_{n}\right\rangle \rightarrow\langle f, \alpha\rangle(f \in \mathscr{S})$. Since $\mathscr{S} \mathscr{S}(\mathscr{A}) \subset \mathscr{S}$, this implies that $\left\langle f \beta, \alpha_{n}\right\rangle \rightarrow\langle f \beta, \alpha\rangle$ $(\beta \in \mathscr{S}(\mathscr{A}), f \in \mathscr{S})$. Hence $\beta \alpha_{n} \rightarrow \beta \alpha$ with respect to $\mathscr{V}$ for all $\beta \in \mathscr{S}(\mathscr{A})$.

3. Let $N \subset \mathbb{S}(\mathscr{A}), \alpha \in \mathscr{V}$-closure of $N$, and $f \in \mathscr{S}$. Then by 2.3, 1 and 1.2, 4, $f \alpha \leqq f^{N}=f^{N \omega} \leqq f^{N} \omega$; whence $\alpha \in \operatorname{cls} \mathscr{S} N$. Thus the identity map of $(\mathscr{S}(\mathscr{A}), \mathscr{V})$ into $(\mathscr{S}(\mathscr{A}), \tau(\mathscr{S}))$ is continuous.

4. Let $U=[(\alpha, \beta) \mid \alpha, \beta \in \mathscr{S}(\mathscr{A})$ and $\langle f, \alpha\rangle=\langle f, \beta\rangle(f \in \mathscr{S})]$. Then $U$ is a $\mathscr{V}$-closed equivalence relation on $\mathscr{S}(\mathscr{A})$. Hence $(\mathscr{S}(\mathscr{A}) / U, \mathscr{V})$ is Hausdorff. But $(\alpha, \beta) \in U$ if and only if $\alpha \beta^{-1} \in \mathbb{S}(\mathscr{S})$ since $\mathscr{S}$ is $T$-invariant.

4.23 LEMMA. $(\mathscr{S}(\mathscr{A}) / \mathscr{S}(\mathscr{S}), \tau(\mathscr{S})$ ) is a Baire space (i.e. the intersection of countably many everywhere dense open sets is again an everywhere dense set).

Proof. Let $\left(A_{n}\right)$ be a sequence of $\tau(\mathscr{S})$-closed subsets of $\mathscr{S}(\mathscr{A}) / \mathscr{S}(\mathscr{S})$ with $\cup A_{n} \supset V$ with $V \tau(\mathscr{S})$-open to show that there exists $n$ with the $\tau(\mathscr{S})$-interior of $A_{n}$ not empty.

By 4.22 (3), $A_{k}$ is $\mathscr{V}$-closed for all $k$, and $V$ is $\mathscr{V}$-open. Hence by 4.22 (4) there exists $n$ with the $\mathscr{V}$-interior of $A_{n}$ not empty.

Now $\mathbb{S}(\mathscr{A}) / \mathscr{S}(\mathscr{S})$ is a group and 4.22 (2) shows that left multiplication by any element is a homeomorphism of $(\mathbb{S}(\mathscr{A}) / \mathscr{S}(\mathscr{S}), \mathscr{V})$ onto $(\mathbb{S}(\mathscr{A}), \mathbb{S}(\mathscr{S}), \mathscr{V})$. Since $(\mathbb{S}(\mathscr{A}) / \mathscr{S}(\mathscr{S}), \mathscr{V})$ is compact, there exists a finite subset $F$ of $\mathscr{S}(\mathscr{A}) / \mathscr{S}(\mathscr{S})$ with $F A_{n}=\mathscr{S}(\mathscr{A}) / \mathscr{S}(\mathscr{S})$.

Finally since left multiplication by any element is a homeomorphism of $(\mathscr{S}(\mathscr{A}) / \mathscr{S}(\mathscr{S}), \tau(\mathscr{S}))$ onto $(\mathscr{S}(\mathscr{A}) / \mathscr{S}(\mathscr{S}), \tau(\mathscr{S}))$ and $A_{n}$ is $\tau(\mathscr{S})$-closed, we may conclude that the $\tau(\mathscr{S})$-interior of $A_{n}$ is not empty. 
4.24 LeMma. 1. $Q \mathscr{S}(\mathscr{B})$ is a $\tau(\mathscr{S})$-closed subgroup of $\mathscr{S}(\mathscr{A})$.

2. If $\mathscr{B}$ is quasi-separable then $Q \mathscr{S}(\mathscr{B}) \neq \mathscr{S}(\mathscr{A})$.

Proof. 1. Let $\eta$ be the canonical map of $\mathscr{S}(\mathscr{A})$ onto $\mathscr{G}(\mathscr{A}) / Q$. Now $\mathscr{S}(\mathscr{B})$ is a $\tau(\mathscr{S})$-compact subgroup of $\mathbb{S}(\mathscr{A})$. Hence $\eta(\mathscr{S}(\mathscr{B}))$ is a $\tau(\mathscr{S})$-compact subgroup of $\mathscr{G}(\mathscr{A}) / Q$. Since $(\mathscr{S}(\mathscr{A}) / Q, \tau(\mathscr{S}))$ is Hausdorff, $\eta(\mathscr{S}(\mathscr{B}))$ is $\tau(\mathscr{S})$-closed. Hence $Q \mathscr{S}(\mathscr{B})=\eta^{-1} \eta(\mathbb{S}(\mathscr{B}))$ is $\tau(\mathscr{S})$-closed.

2. Assume that $\mathscr{B}$ is quasi-separable and that $Q \mathscr{S}(\mathscr{B})=\mathscr{S}(\mathscr{A})$. Let $f \in \mathscr{B},\{f\}$ the $T$-subalgebra of $\mathscr{B}$ generated by $f, \mathscr{H}$ a countable, dense subset of $\{f\}$, and $F(\mathscr{H})$ the collection of finite subsets of $\mathscr{H}$. For each $L \in F(\mathscr{H})$ let $L$ again denote the map $\alpha \rightarrow \inf \left[\sum_{h \in L}|\langle h, \alpha t\rangle-\langle h, t\rangle| \mid t \in T\right]$ of $\mathscr{S}(\mathscr{A})$ into $R$. Let $\chi$ denote the canonical map of $\&(\mathscr{A})$ onto $\mathscr{S}(\mathscr{A}) / \mathscr{S}(\mathscr{S})$.

Then each $L$ in $F(\mathscr{H})$ induces a map $\mathcal{L}$ of $\mathscr{S}(\mathscr{A}) / \mathscr{S}(\mathscr{S})$ into $R$; namely $\langle\mathcal{L} ; \chi(\alpha)\rangle$ $=\langle L, \alpha\rangle(\alpha \in \mathbb{S}(\mathscr{A}))$. Let $\hat{C}_{L}$ denote the points at which $\mathcal{L}$ is continuous and $C_{L}$ those at which $L$ is continuous.

Now $\chi^{-1} \mathcal{L}^{-1}[a, \infty)=L^{-1}[a, \infty)$ is a $\tau(\mathscr{S})$-closed subset of $\mathscr{S}(\mathscr{A})$ for all real numbers $a$, by 4.18. Hence $\mathcal{L}^{-1}[a, \infty)$ is a $\tau(\mathscr{S})$-closed subset of $\mathscr{S}(\mathscr{A}) / \mathscr{S}(\mathscr{S})$ whence $\mathcal{L}$ is upper semicontinuous. Hence $\hat{C}=\cap\left[\hat{C}_{L} \mid L \in F(\mathscr{H})\right]$ is a residual subset of $(\mathscr{S}(\mathscr{A}) / \mathscr{S}(\mathscr{S}), \tau(\mathscr{S}))$ whence by $4.23 \hat{C}$ is a $\tau(\mathscr{S})$-dense set.

It is clear that $\chi^{-1}\left(\hat{C}_{L}\right) \subset C_{L}(L \in F(\mathscr{H}))$ and that $\chi^{-1}(\hat{C}) \subset C=\cap\left[C_{L} \mid L \in F(\mathscr{H})\right]$. Since $\chi$ is an open mapping, $C$ is $\tau(\mathscr{S})$-dense in $\mathscr{S}(\mathscr{A})$.

Lemma 4.21 together with the assumption that $Q \mathscr{S}(\mathscr{B})=\mathscr{S}(\mathscr{A})$ implies that $f \alpha=f(\alpha \in C)$. But the set of $\alpha$ for which $f \alpha=f$ is $\tau(\mathscr{S})$-closed. Thus $f \alpha=f$ for all $\alpha \in \operatorname{cls} \mathscr{S} C=\mathscr{B}(\mathscr{A})$. Since $f$ was an arbitrary element of $\mathscr{B}$, this implies that $\mathscr{S}(\mathscr{A})$ $\subset \mathfrak{S}(\mathscr{B})$. Hence $\mathbb{S}(\mathscr{A})=\mathscr{S}(\mathscr{B})$ whence $\mathscr{B}=\mathscr{A}$, a contradiction. The proof is completed.

4.25 Proposition. Let $\mathscr{A}, \mathscr{B}$ be T-subalgebras of $\mathscr{M}$ such that $\mathscr{A} \subset \mathscr{B}$ and such that $\mathscr{B}$ is a nontrivial, quasi-separable, group-like extension of $\mathscr{A}$. Then:

(1) There exists a nontrivial isometric extension $\mathscr{W}$ of $\mathscr{A}$ with $\mathscr{A} \subset \mathscr{W} \subset \mathscr{B}$.

(2) There exist an ordinal $\nu$ and a collection of T-subalgebras $\left(\mathscr{B}_{\alpha} \mid \alpha \leqq \nu\right)$ such that (I) $\mathscr{B}_{0}=\mathscr{A}$, (II) $\mathscr{B}_{\nu}=\mathscr{B}$, (III) $\mathscr{B}_{\alpha} \subset \mathscr{B}_{\beta}(\alpha \leqq \beta \leqq \nu)$, (IV) $\mathscr{B}_{\alpha+1}$ is a nontrivial isometric extension of $\mathscr{B}_{\alpha}(\alpha<\nu)$, and $(\mathrm{V}) \mathscr{B}_{\alpha}=\operatorname{cl}\left(\cup\left[\mathscr{B}_{\beta} \mid \beta<\alpha\right]\right)$ for all limit ordinals $\alpha \leqq \nu$.

Proof. 1. Set $\mathscr{Q}=[f \mid f \in \mathscr{S}, f \alpha=f,(\alpha=Q)]$ and

$$
\mathscr{H}=[f \mid f \in \mathscr{S}, f \alpha=f,(\alpha \in Q \mathscr{S}(\mathscr{B}))] .
$$

Then $\mathscr{A} \subset \mathscr{H}$ and $\mathscr{A} \neq \mathscr{H}$ by Lemmas 4.24 and 3.9. Moreover $\mathscr{A} \subset \mathscr{H} \subset \mathscr{Q}$ and $\mathscr{2}$ is a principal extension of $\mathscr{A}$ by 4.17 .

Proposition 4.25 (I) now follows from 4.8 with $|\mathscr{Q}|=X,|\mathscr{H}|=Z,|\mathscr{A}|=Y$. The algebra $\mathscr{W}$ corresponding to $W$ will be contained in $\mathscr{H}$ which in turn is contained in $\mathscr{B}$.

2. Set $\mathscr{B}_{0}=\mathscr{A}$. Assume $\mathscr{B}_{\alpha}$ defined for all ordinals $\alpha$ less than a fixed ordinal $\gamma$ 
such that the family $\left(\mathscr{B}_{\alpha} \mid \alpha<\gamma\right)$ has properties (I), (III), (IV), (V) of Proposition 4.25 where $\nu$ is replaced by $\gamma$.

I wish to define $\mathscr{B}_{\gamma}$. If $\gamma$ is a limit ordinal set $\mathscr{B}_{\gamma}=\operatorname{cl}\left(\bigcup\left[\mathscr{B}_{\alpha} \mid \alpha<\gamma\right]\right)$. Otherwise, suppose $\gamma=\beta+1$. If $\mathscr{B}_{\beta}=\mathscr{B}$, set $\mathscr{B}_{\gamma}=\mathscr{B}$. Otherwise choose $\mathscr{B}_{\gamma}$ to be a nontrivial isometric extension of $\mathscr{B}_{\beta}$ with $\mathscr{B}_{\gamma} \subset \mathscr{B}$. (This is possible by 1.)

Thus $\mathscr{B}_{\alpha}$ is defined for all ordinals $\alpha$. Since $\mathscr{B}_{\alpha} \subset \mathscr{B}$ for all $\alpha$ and $\mathscr{B}_{\alpha} \nsupseteq \mathscr{B}_{\beta}$ if $\alpha>\beta$ unless $\mathscr{B}_{\beta}=\mathscr{B}$, there must be a smallest ordinal $\nu$ such that $\mathscr{B}_{\nu}=\mathscr{B}$. Then it is easy to verify that the family $\left(\mathscr{B}_{\alpha} \mid \alpha \leqq \nu\right)$ has the desired properties.

The proof of the following structure theorem is similar to the proof of 4.25 and will be omitted.

4.26 Proposition. Let $\mathscr{A}, \mathscr{S}$ be $T$-subalgebras of $\mathscr{M}$ such that $\mathscr{A} \subset \mathscr{S}$ and such that $\mathscr{S}$ is a nontrivial, quasi-separable, group extension of $\mathscr{A}$. Then (1) there exists a nontrivial principal extension $\mathscr{G}$ of $\mathscr{A}$ such that $\mathscr{A} \subset \mathscr{G} \subset \mathscr{S}$ and such that the group of the extension is a compact Lie group. (2) There exist an ordinal $v$ and a family of $T$-subalgebras $\left(\mathscr{S}_{\alpha} \mid \alpha \leqq v\right)$ such that (i) $\mathscr{S}_{0}=\mathscr{A}$, (ii) $\mathscr{S}_{\nu}=\mathscr{S}$ (iii) $\mathscr{S}_{\alpha} \subset \mathscr{S}_{\beta}(\alpha \leqq \beta \leqq \nu$ ), (iv) $\mathscr{S}_{\alpha+1}$ is a nontrivial principal extension of $\mathscr{S}_{\alpha}$ such that the group of the extension is a compact Lie group, (v) $\mathscr{S}_{\alpha}=\operatorname{cl}\left(\bigcup\left[\mathscr{S}_{\beta} \mid \beta<\alpha\right]\right)$ for all limit ordinals $\alpha \leqq \nu$.

In Proposition $4.26 \mathscr{S}_{\alpha+1}$ is in general not the "largest possible" principal extension of $\mathscr{S}_{\alpha}$, the reason being that it is insisted that the group of the extension be a Lie group. If this is dropped and the principal extension is always taken to be the largest possible, then the various extensions and groups involved may be explicitly described.

Set $\mathscr{G}_{0}=\mathscr{A}, Q_{0}=\mathscr{S}(\mathscr{A}), Q_{1}=Q\left(Q_{0}, \mathscr{S}\right)$ and $\mathscr{G}_{1}=\mathfrak{U}\left(Q_{1}\right) \cap \mathscr{S}$ (i.e. those $f \in \mathscr{S}$ with $f \alpha=f\left(\alpha \in Q_{1}\right)$ ). Then $Q_{1}$ is a $\tau(\mathscr{S})$-closed, invariant subgroup of $Q_{0}$ and under the assumptions of $4.26, \mathscr{G}_{1}$ is a nontrivial principal extension of $\mathscr{G}_{0}$ with group $Q_{0} / Q_{1}$.

By Proposition 3.9, $Q_{1}=\&\left(\mathscr{G}_{1}\right)$ and so we may apply the above considerations to $\mathscr{G}_{1}, Q_{1}, Q_{2}=Q\left(Q_{1}, \mathscr{S}\right)$, and $\mathscr{G}_{2}=\mathfrak{A}\left(Q_{2}\right) \cap \mathscr{S}$ in order to continue the construction.

Let $\beta$ be an ordinal greater than one and suppose we have defined two families $\left(\mathscr{G}_{\alpha} \mid \alpha<\beta\right)$ and $\left(Q_{\alpha} \mid \alpha<\beta\right)$ such that (I) $\mathscr{G}_{\alpha}$ is a $T$-subalgebra of $\mathscr{S}$ and $Q_{\alpha}$ is a $\tau(\mathscr{C})$-closed subgroup of $\mathscr{S}(\mathscr{A})$ for all $\alpha<\beta$, (II) $\mathscr{G}_{\alpha}=\mathscr{S} \cap \mathfrak{X}\left(Q_{\alpha}\right)(\alpha<\beta)$, (III) if $\alpha=\gamma+1$ and $\alpha<\beta$ then $Q_{\alpha}=Q\left(Q_{\gamma}, \mathscr{S}\right)$, (IV) if $\alpha$ is a limit ordinal with $\alpha<\beta$ then $Q_{\alpha}=\bigcap\left[Q_{\gamma} \mid \gamma<\alpha\right]$.

If $\beta$ is a limit ordinal, set $Q_{\beta}=\bigcap\left[Q_{\alpha} \mid \alpha<\beta\right]$ and $\mathscr{G}_{\beta}=\mathfrak{U}\left(Q_{\beta}\right) \cap \mathscr{S}$. If $\beta=\alpha+1$ then set $Q_{\beta}=Q\left(Q_{\alpha}, \mathscr{S}\right)$ and $\mathscr{G}_{\beta}=\mathfrak{X}\left(Q_{\beta}\right) \cap \mathscr{S}$.

Thus we have constructed two families $\left(\mathscr{G}_{\alpha}\right)$ and $\left(Q_{\alpha}\right)$ satisfying (I), (II), (III), and (IV) for all ordinals $\alpha$. If $Q_{\alpha} \neq \mathscr{S}(\mathscr{S})$ then Lemma 4.24 shows that $Q_{\beta} \neq Q_{\alpha}$ for all $\beta<\alpha$. This implies that $\mathscr{G}_{\alpha} \neq \mathscr{S}$ and that $\mathscr{G}_{\alpha}$ is a nontrivial extension of $\mathscr{G}_{\beta}$ for all $\beta<\alpha$. Hence there exists a least ordinal $\nu$ with $Q_{\nu}=\mathbb{S}(\mathscr{S})$ and $\mathscr{G}_{v}=\mathscr{S}$. This completes the proof of the following proposition. (Recall that by $3.9 \mathscr{G}_{\alpha}=\mathfrak{X}\left(Q_{\alpha}\right) \cap \mathscr{S}$ implies that $Q_{\alpha}=\mathbb{S}\left(\mathscr{G}_{\alpha}\right)$.) 
4.27 Proposition. Let $\mathscr{A}, \mathscr{S}$ be $T$-subalgebras of $\mathscr{M}$ such that $\mathscr{A} \subset \mathscr{S}$ and such that $\mathscr{S}$ is a nontrivial, quasi-separable, group extension of $\mathscr{A}$. Then there exist an ordinal $\nu$ and two families $\left(\mathscr{G}_{\alpha} \mid \alpha \leqq v\right),\left(Q_{\alpha} \mid \alpha \leqq \nu\right)$ such that

(I) $\mathscr{G}_{\alpha}$ is a $T$-subalgebra of $\mathscr{S}$ for all $\alpha \leqq \nu$ with $\mathscr{G}_{0}=\mathscr{A}$ and $\mathscr{G}_{\nu}=\mathscr{S}$,

(II) $Q_{\alpha}$ is a $\tau(\mathscr{C})$-closed subgroup of $\mathbb{S}(\mathscr{A})$ with $Q_{0}=\mathscr{S}(\mathscr{A})$ and $Q_{v}=\mathbb{S}(\mathscr{S})$,

(III) $\mathscr{G}_{\alpha}=\mathscr{S} \cap \mathfrak{A}\left(Q_{\alpha}\right)$ and $Q_{\alpha}=\mathbb{S}\left(\mathscr{G}_{\alpha}\right)(\alpha \leqq \nu)$,

(IV) $Q_{\alpha+1}=Q\left(Q_{\alpha}, \mathscr{F}\right)(\alpha+1 \leqq \nu)$ and $Q_{\beta}=\bigcap\left[Q_{\gamma} \mid \gamma<\beta\right]$ for all limit ordinals $\beta \leqq \nu$.

(V) $Q_{\alpha} \supset Q_{\beta}(\alpha \leqq \beta \leqq \nu)$ and $Q_{\alpha+1}$ is a $\tau(\mathscr{S})$-closed invariant subgroup of $Q_{\alpha}$ $(\alpha+1 \leqq \nu)$,

(VI) $\mathscr{G}_{\alpha} \subset \mathscr{G}_{\beta}(\alpha \leqq \beta \leqq \nu)$ and $\mathscr{G}_{\alpha+1}$ is a principal extension of $\mathscr{G}_{\alpha}$ with group $Q_{\alpha} / Q_{\alpha+1}(\alpha+1 \leqq \nu)$.

4.28 Remarks. 1. Using the notation of Proposition 4.26 let $\alpha$ be a limit ordinal with $\alpha \leqq \nu$. Set $\mathscr{H}=\operatorname{cl}\left(\bigcup\left[\mathscr{G}_{\beta} \mid \beta<\alpha\right]\right)$. Then $\mathscr{H}$ is a $T$-subalgebra of $\mathscr{S}$. A simple verification shows that $\&(\mathscr{H})=Q_{\alpha}$. Hence $\mathscr{H}=\mathscr{G}_{\alpha}$ by 3.9 .

2. Proposition 4.25 is indeed a generalization of the Furstenberg structure theorem (Proposition 3.13) since the latter is obtained from 4.25 by setting $\mathscr{A}$ equal to the algebra of constant functions.

5. In this section I would like to apply the main theorem to the case where the algebra under consideration is the algebra of distal functions. Throughout this section $\mathscr{D}$ will denote the $T$-subalgebra of $\mathscr{C}$ consisting of all distal functions and $\mathscr{E}$ the $T$-subalgebra of $\mathscr{C}$ consisting of all almost periodic functions. Recall that $f \in \mathscr{E}$ if and only if $[t f \mid t \in T]$ is a relatively compact subset of $\mathscr{C}$.

5.1 LeMma. 1. $f p \in \mathscr{E}$ for all $f \in \mathscr{E}$ and $p \in \beta T$.

2. $\langle f, e\rangle=\langle f, v\rangle$ for all $f \in \mathscr{E}$ and for all idempotents $v$.

3. $\mathscr{E} \subset \mathscr{D}$ i.e. $f p v=f p\left(f \in \mathscr{E}, p \in \beta T, v^{2}=v \in M\right)$.

Proof. 1. Let $f \in \mathscr{E}, p \in \beta T$. If the net of functions $\left(t_{n} f\right)$ converges uniformly to $h$ then the net $\left(t_{n} f p\right)$ converges uniformly to $h p$. Hence $f p \in \mathscr{E}$.

2. Let $v^{2}=v \in \beta T$. Choose a net $\left(t_{n}\right)$ of elements of $T$ such that $t_{n} \rightarrow v$ and suppose that $t_{n}^{-1} \rightarrow p \in \beta T$.

Let $f \in \mathscr{E}$. Then the functions $\left(t_{n}^{-1} f\right)$ converge pointwise to the function $p f$. Since $f \in \mathscr{E},\left(t_{n}^{-1} f\right)$ converges uniformly to $p f$. Let $\varepsilon>0$. Then there exists $n_{0}$ such that $\left|\left\langle t_{n}^{-1} f, q\right\rangle-\langle p f, q\rangle\right|\left\langle\varepsilon(q \in \beta T, n\rangle n_{0}\right)$. Now set $q=r t_{n}$. Then $\mid\langle f, r\rangle-$ $\left\langle f, r t_{n} p\right\rangle \mid\left\langle\varepsilon(r \in \beta T, n\rangle n_{0}\right)$. Hence we may conclude that $\langle f, r\rangle=\langle f, r v p\rangle(r \in \beta T)$. When $r=e$ this gives $\langle f, e\rangle=\langle f, v p\rangle$ and when $r=v$ that $\langle f, v\rangle=\left\langle f, v^{2} p\right\rangle=\langle f, v p\rangle$. Thus $\langle f, e\rangle=\langle f, v\rangle$.

3. Let $f \in \mathscr{E}, p \in \beta T$ and $v^{2}=v$. Let $t \in T$. Then $t f p \in \mathscr{E}$ by 1 and the fact that $\mathscr{E}$ is a $T$-subalgebra of $\mathscr{C}$. By $2\langle t f p, e\rangle=\langle t f p, v\rangle$; i.e. $\langle f p, t\rangle=\langle f p v, t\rangle$. Since this is true for all $t \in T, f p=f p v$. 
5.2 Lemma. Let $f \in \mathscr{E}$. Then

1. $\left\langle f^{N}, p\right\rangle=\sup [\langle f q, p\rangle \mid q \in N]$ for all subsets $N$ of $\beta T$ and all $p \in \beta T$.

2. $\left\langle f_{N} ; p\right\rangle=\inf [\langle f q, p\rangle \mid q \in N]$ for all subsets $N$ of $\beta T$ and all $p \in \beta T$.

3. If $f \in \mathscr{A}, a$-subalgebra of $\mathscr{C}$, then the maps $\alpha \rightarrow\langle f, \alpha p\rangle$ of $(G, \tau(\mathscr{A}))$ into $R$ are continuous for all $p \in \beta T$.

Proof. 1. Let $\varepsilon>0, p \in \beta T$. Since $f^{N}$ is continuous and $f \in \mathscr{E}$, there exists $t \in T$ such that $\left|\left\langle f^{N}, t\right\rangle-\left\langle f^{N}, p\right\rangle\right|\left\langle\varepsilon\right.$ and $|\langle f, q t-f, q p\rangle|\left\langle\varepsilon(q \in \beta T)\right.$. Then $\left\langle f^{N}, p\right\rangle-\varepsilon$ $\left\langle\left\langle f^{N}, t\right\rangle=\sup [\langle f, q t\rangle \mid q \in N]\langle\sup [\langle f, q p\rangle \mid q \in N]+\varepsilon\right.$. Thus

$$
\left\langle f^{N}, p\right\rangle \leqq \sup [\langle f, q p\rangle \mid q \in N]
$$

and a similar argument shows that $\sup [\langle f, q p\rangle \mid q \in N] \leqq\left\langle f^{N}, p\right\rangle$.

2. The proof of 2 is similar to that of 1 and will be omitted.

3. Let $p \in \beta T,\langle h, \alpha\rangle=\langle f, \alpha p\rangle(\alpha \in G), a, b$ real numbers with $a\langle b$. I shall show that $h^{-1}(a, b)$ is $\tau(\mathscr{A})$-open or equivalently that $h^{-1}[b, \infty)$ and $h^{-1}(-\infty, a]$ are $\tau(\mathscr{A})$-closed.

Let $K=h^{-1}(-\infty, a]$ and $\alpha \in \operatorname{cls}_{\mathscr{A}} K$. Since $f \in \mathscr{A}, f \alpha \leqq f^{K} \omega$. Hence $\langle f, \alpha p\rangle=$ $\langle f \alpha, p\rangle \leqq\left\langle f^{K} \omega, p\right\rangle=\left\langle f^{K}, \omega p\right\rangle=\sup [\langle f \beta, \omega p\rangle \mid \beta \in K]=\sup [\langle f, \beta p\rangle \mid \beta \in K] \leqq a$ (by the definition of $K$ ). Hence $\alpha \in K$, whence $K$ is $\tau(\mathscr{A})$-closed. A similar argument using 2 instead of 1 shows that $h^{-1}[b, \infty)$ is also closed.

5.3 Proposition. Let $\mathscr{A}$ be a T-subalgebra of $\mathscr{C}$ such that $\mathscr{A} G \subset \mathscr{A}$. Then $\mathscr{A} \subset \mathscr{E}$ if and only if $(G / \mathscr{S}(\mathscr{A}),|\mathscr{A}|, T)$ is a principal extension of the trivial minimal set with group $G / \mathscr{S}(\mathscr{A})$.

Proof. Assume $\mathscr{A} \subset \mathscr{E}$. Now apply Proposition 4.14 with $\mathscr{F}=\mathscr{A}$ and $K=G$. Then Lemma 5.2 shows that $\mathscr{L}=\mathscr{A}$. Thus all that remains to be shown is that $\mathfrak{A}(G) \cap \mathscr{A}=R$.

Let $f \in \mathfrak{A}(G) \cap \mathscr{A}, x \in \beta T$. Then $\langle f, x\rangle=\langle f u, x\rangle$ by 5.1. Furthermore $\langle f u, x\rangle$ $=\langle f u x, e\rangle=\langle f u x u, e\rangle$ again by 5.1. But $u x u \in G$ and $f \in \mathfrak{X}(G)$. Hence $\langle f u x u, e\rangle$ $=\langle f, e\rangle$, whence $\langle f, x\rangle=\langle f, e\rangle$ and $f \in R$.

Now suppose that $(G / \mathscr{S}(\mathscr{A}),|\mathscr{A}|, T)$ is a principal extension of the trivial minimal set with group $G / \mathscr{S}(\mathscr{A})$. Let $\left(t_{n}\right)$ be a net of elements of $T$ which converges pointwise to $p \in \beta T$, and let $f \in \mathscr{A}$. I wish to show that the functions $\left(t_{n} f\right)$ converge uniformly to the function $p f$.

Let $\Pi: G / \mathscr{S}(\mathscr{A}) \times|\mathscr{A}| \rightarrow|\mathscr{A}|$ be the map such that $\Pi(\alpha \mathscr{S}(\mathscr{A}), x)=\alpha x(x \in|\mathscr{A}|$, $\alpha \in G)$. Let $\varepsilon>0$ and $\eta$ the index on $|\mathscr{A}|$ defined by

$$
\eta=[(x, y)|x, y \in| \mathscr{A}|,|\langle f, x\rangle-\langle f, y\rangle \mid<\varepsilon] .
$$

Then since $\Pi$ is continuous and the spaces involved are compact Hausdorff, there exists a neighborhood $N$ of $p \mid \mathscr{A}$ such that $(\alpha x, \alpha y) \in \eta$ for all $\alpha \in G$ and all $x, y \in N$. There exists $n_{0}$ such that $t_{n} \in N\left(n \geqq n_{0}\right)$. Thus

$$
\left|\left\langle f, \alpha t_{n}\right\rangle-\left\langle f, \alpha t_{m}\right\rangle\right|<\varepsilon \quad\left(n, m \geqq n_{0}, \alpha \in G\right) .
$$


Now $|R|$ is trivial and $\mathscr{A}$ is a group-like extension of $R$. Hence given $q \in \beta T$ there exists $\alpha \in G$ with $\alpha|\mathscr{A}=q| \mathscr{A}$. Since $t f \in \mathscr{A}$ for all $t \in T$, we may conclude that $\left|\left\langle t_{n} f, q\right\rangle-\left\langle t_{m} f, q\right\rangle\right|<\varepsilon\left(n, m \leqq n_{0}, q \in \beta T\right)$. Thus the net $\left(t_{n} f\right)$ is uniformly Cauchy and since it converges pointwise to $p f$, it converges uniformly to $p f$. The proof is completed.

5.4 Proposition. 1. $\mathscr{D} G \subset \mathscr{D}$.

2. $\mathscr{E}=\mathfrak{Q}(Q) \cap \mathscr{D}$, where $Q=Q(G, \mathscr{D})$.

3. $\mathbb{B}(\mathscr{E})=Q(G, \mathscr{D})$.

4. $(G / \mathbb{S}(\mathscr{E}), \tau(\mathscr{D}))$ is the Bohr compactification of the discrete group $T$.

Proof. 1. Let $f \in \mathscr{D}$. Then $f p v=f p\left(p \in \beta T, v^{2}=v \in M\right)$. Replacing $p$ by $\alpha p$ in the above relation gives $f \alpha p v=f \alpha p\left(p \in \beta T, v^{2}=v \in M\right)$. Thus $f \alpha \in \mathscr{D}(\alpha \in G)$.

2. Let us apply Proposition 4.14 with $\mathscr{F}=\mathscr{D}$ and $K=G$. Then as in the proof of $5.3 \mathfrak{A}(G) \cap \mathscr{D}=R$. Thus the $T$-subalgebra $\mathscr{W}=[f \mid f \in \mathscr{D}, \alpha \rightarrow\langle f, \alpha p\rangle$ of $(G, \tau(\mathscr{D}))$ into $R$ is continuous for all $p \in \beta T$ ] is a principal extension of $R$ with group $G / \mathbb{S}(\mathscr{W})$. Hence $\mathscr{W} \subset \mathscr{E}$ by 5.3. But $\mathscr{E} \subset \mathscr{W}$ by 5.2 since $\mathscr{E} \subset \mathscr{D}$ by 5.1. Thus $\mathscr{E}=\mathscr{W}$.

Now $\mathscr{D}$ is a group-extension of $R$. Then by Proposition 4.17 and the discussion preceding it $\mathscr{E}=\mathscr{U}(Q) \cap \mathscr{D}$.

3. This follows from 2, the fact that $\mathscr{D}$ is a group-like extension of $R \subset \mathscr{E}$, and Proposition 3.9.

4. Let $\eta: T \rightarrow G$ be the map such that $\eta(t)=u t u(t \in T)$, $\Pi$ the canonical map of $G$ onto $G / \mathscr{S}(\mathscr{E})$, and $\sigma=\pi \eta$.

Let $f \in \mathscr{E}, t, s \in T$. Then the equations futs ${ }^{-1} u u s u=f u t s^{-1} u s u=\left(f u t s^{-1} u\right) s u$ $=\left(\right.$ futs $\left.{ }^{-1}\right) s u=f u t u$ show that $\eta\left(t s^{-1}\right) \eta(s)$ is congruent to $\eta(t)$ modulo $\&(\mathscr{E})$. Hence $\sigma$ is a homomorphism of $T$ into $G / \mathscr{S}(\mathscr{E})$.

Now let $\alpha \in G$. Choose a net $\left(t_{n}\right)$ of elements of $T$ which converges pointwise to $\alpha$. Let $f \in \mathscr{D}$ and $t \in T$. Then $t_{n} t$ converges pointwise to $\alpha t$. Hence $\langle f \alpha, t\rangle=\langle f, \alpha t\rangle$ $=\lim \left\langle f, t_{n} t\right\rangle=\lim \left\langle f u t_{n} u, t\right\rangle$ since $f=f u$ and $f u t_{n}=f u t_{n} u$. Thus $\langle f \alpha, t\rangle \leqq\left\langle f^{K}, t\right\rangle$ $(t \in T)$ where $K=\eta(T)$. This implies that $f \alpha \leqq f^{\mathbb{R}}=f^{\mathbb{u}} \leqq f^{\mathbb{R}} u$. Hence clsg $\eta(T)=G$ and $\sigma(T)$ is dense in $(G / \mathscr{S}(\mathscr{E}), \tau(\mathscr{D}))$.

Now let $H$ be a compact topological group and $\delta$ a homomorphism of $T$ into $H$ with dense range. Then there exists a continuous extension $\rho$ of $\delta$ to $\beta T$. It is easy to verify that $\rho(x y)=\rho(x) \rho(y)(x, y \in \beta T)$.

Since $H$ is a compact topological group $\rho^{*}(\mathscr{C}(H)) \subset \mathscr{E}$. Thus $\rho$ induces a continuous homomorphism $\bar{\rho}$ of $(G / \mathbb{S}(\mathscr{E}), \mathscr{V})$ onto $H$ where $\mathscr{V}$ is the quotient topology induced by the topology of pointwise convergence on $G$.

By Lemma 4.22 with $\mathscr{A}=R$ and $\mathscr{S}=\mathscr{E}$ we see that $\mathscr{V}$ is the same as that induced by $\tau(\mathscr{E})$. Since $\mathscr{E} \subset \mathscr{D}, \tau(\mathscr{E}) \subset \tau(\mathscr{D})$ and because $(G / \mathbb{S}(\mathscr{E}), \tau(\mathscr{D}))$ is compact $\tau(\mathscr{D})$ induces the same topology on $G / \mathbb{S}(\mathscr{E})$ as does $\tau(\mathscr{E})$.

Finally it is immediate that $\delta(t)=\rho(u t u)=\bar{\rho}(\sigma(t))$. The proof is completed.

5.5 REMARK. It is also of interest to apply the main proposition (4.14) to the case $\mathscr{F}=\mathscr{M}=\mathscr{X}(\omega)$ and $K=G$. The situation is complicated in this instance by the 
fact that $\mathfrak{X}(G) \cap \mathscr{M}$ need not be $R$. (See for example [10] where it is shown that $\mathfrak{U}(G) \cap \mathscr{M} \neq R$ when $T$ is the group of homeomorphisms of the circle.)

If we assume, however, that $\mathfrak{R}(G) \cap \mathscr{M}=R$ then $\mathscr{L}$ of Proposition 4.14 turns out to be $\mathscr{E}$. The reason for this is that $\mathscr{L} \subset \mathscr{E}$ by Proposition 5.3 and $\mathscr{E} \subset \mathscr{L}$ by Lemma 5.2.

Since $\mathscr{D} \subset \mathscr{M}, \tau(\mathscr{D}) \subset \tau(\mathscr{M})$ and Proposition 5.4 implies that $(G / \mathscr{S}(\mathscr{E}), \tau(\mathscr{M}))$ is the Bohr compactification of the discrete group $T$. (Recall that $(G, \tau(\mathscr{M})$ ) is compact.)

An important instance when $\mathfrak{A}(G) \cap \mathscr{M}=R$ is when $T$ is abelian. To see this observe that in this case $u t=u t u \in G(t \in T)$. Thus if $f \in \mathfrak{X}(G) \cap \mathscr{M}$ and $t \in T$, $\langle f, t\rangle=\langle f t, e\rangle$; but $f t=f u t=f$, whence $\langle f, t\rangle=\langle f, e\rangle$.

5.6 REMARK. Another point worth considering is the relation between $Q(G, \mathscr{M})$ and $\mathscr{S}(\mathscr{E})$. Since $\mathscr{D} \subset \mathscr{M}, Q(G, \mathscr{M}) \subset Q(G, \mathscr{D})$ and so $Q(G, \mathscr{M}) \subset \mathcal{G}(\mathscr{E})$ by Proposition 5.4. I do not know whether in general $Q(G, \mathscr{M})=\mathbb{E}(\mathscr{E})$. If this be not the case it would be interesting to identify the compact topological group $(G / Q(G, \mathscr{M}), \tau(\mathscr{M}))$ which is "bigger" than the Bohr compactification of $T$ and which is associated with $T$ in a natural fashion.

When $T$ is abelian $Q(G, \mathscr{M})=\mathbb{S}(\mathscr{E})$. To see this let $\eta: T \rightarrow G$ be the map such that $\eta(t)=u t u=u t, \Pi, \chi$ the canonical maps of $G$ onto $G / \mathbb{S}(\mathscr{E})$ and $G / Q(G, \mathscr{M})$ respectively. Set $\sigma=\Pi \eta$ and $\rho=\chi \eta$.

Since $T$ is abelian, $\eta$ is a homomorphism of $T$ into $G$. Hence $\sigma$ and $\rho$ are homomorphisms of $T$ into $G / \mathscr{S}(\mathscr{E})$ and $G / Q(G, \mathscr{M})$ respectively.

The identity map of $G$ induces a continuous homomorphism $\phi$ of $(G / Q(G, \mathscr{M}), \tau(\mathscr{M}))$ onto $(G / \mathbb{S}(\mathscr{E}), \tau(\mathscr{M}))$ such that $\phi \rho=\sigma$. Since $(G / \mathbb{S}(\mathscr{E}), \tau(\mathscr{M}))$ together with $\sigma$ is the Bohr compactification of $T$ by 5.4 and 5.5, all that remains to be shown is that $\rho(T)$ is dense in $(G / Q(G, \mathscr{M}), \tau(\mathscr{M}))$. Now one shows this by proving that $\operatorname{cls}_{\mathscr{M}} \eta(T)=G$ just as in the proof of 4 of 5.4 .

6. In this section I show that the results obtained previously depend "naturally" on the minimal ideal $M$ and the idempotent $u$ in $M$. In order to be precise I shall employ the language of category theory.

6.1 Definitions and notation. $J$ will denote the set of idempotents of $\beta T$ which belong to some minimal subset of $\beta T$.

Let $v \in J$ and $N$ the minimal subset of $\beta T$ with $v \in N$. Then $K(v)$ will denote the category whose objects are $T$-subalgebras $\mathscr{A}$ of $\mathscr{C}$ with $\mathscr{A} \subset \mathfrak{U}(v)=[f \mid f \in \mathscr{C}, f v=f]$.

Let $\mathscr{A}, \mathscr{B}$ be objects of $K(v)$. Then the set of morphisms from $\mathscr{A}$ to $\mathscr{B}$ $(\operatorname{Hom}(\mathscr{A}, \mathscr{B}))$ is just the set of $T$-homomorphisms from $\mathscr{A}$ to $\mathscr{B}$.

6.2 REMARKs. 1. Let $v \in J, N$ the minimal subset of $\beta T$ with $v \in N, \mathscr{A}, \mathscr{B}$ objects of $K(v)$ and $\phi \in \operatorname{Hom}(\mathscr{A}, \mathscr{B})$. Then we know that there exists $p \in \beta T$ with $p \mid \mathscr{A}=\phi$, i.e. $\phi(f)=f p(f \in \mathscr{A})$. Since $\mathscr{A} \subset \mathfrak{X}(v), p|\mathscr{A}=v p| \mathscr{A}$. Moreover, since $\phi(\mathscr{A}) \subset \mathscr{B}$, $\mathscr{A} p \subset \mathscr{B} \subset \mathfrak{A}(v)$ and $v p|\mathscr{A}=v p v| \mathscr{A}$. Thus we may conclude that

$$
\operatorname{Hom}(\mathscr{A}, \mathscr{B})=[p|\mathscr{A}| p \in N v \text { and } \mathscr{A} p \subset \mathscr{B}] \text {. }
$$


2. If we take $v=u$ in 1 , then $N$ becomes $M$ and the objects of $K(u)$ are nothing but the $T$-subalgebras of $\mathscr{M}$ considered in the previous sections.

Moreover, $M u=G$ so that in this case $\operatorname{Hom}(\mathscr{A}, \mathscr{B})=[\alpha|\mathscr{A}| \alpha \in G, \mathscr{A} \alpha \subset \mathscr{B}]$.

6.3 Proposition. Let $N$ and $L$ be minimal subsets of $\beta T, v, w \in N \cap J, \delta, \gamma \in L \cap J$ such that $v \gamma=v, \gamma v=\gamma, w \delta=w, \delta w=\delta$. Then the covariant functor $F$ such that $F(\mathscr{A})=\mathscr{A} \delta$ for all objects $\mathscr{A}$ of $K(v)$ and $F(p)=\gamma p \delta(p \in \operatorname{Hom}(\mathscr{A}, \mathscr{B}) \in K(v))$ is a natural equivalence of $K(v)$ with $K(\delta)$, its inverse being the functor $G$ where $G(\mathscr{L})=\mathscr{L} v$ and $G(q)=$ wqv for all objects $\mathscr{L}$ of $K(\delta)$ and all morphisms $q$ of $K(\delta)$.

Proof. Let $p: \mathscr{A} \rightarrow \mathscr{B}, q: \mathscr{B} \rightarrow \mathscr{L}$ be elements of $K(v)$. Then $F(p q)=\gamma p q \delta$ $=\gamma p \gamma q \delta=\gamma p \delta \gamma q \delta=F(p) F(q)$ since as remarked above we may assume that $p v=p$ whence $p \gamma=p$. (Recall that $\delta \gamma=\gamma$ since they are both in the same minimal set.)

The natural equivalence $\sigma: I \rightarrow G F$ is given by $\sigma(\mathscr{A})(f)=f v \delta v=f \delta v \in G F(\mathscr{A})$ $=\mathscr{A} \delta v$ for all objects of $K(v)$, and $f \in \mathscr{A}$. To see that this is so let $p: \mathscr{A} \rightarrow \mathscr{B}$ be an element of $K(v)$, and $f \in \mathscr{A}$. Then $(G F)(p) \sigma(\mathscr{A})(f)=(G F)(p)(f \delta v)$. Now $(G F)(p)$ is the map $h \rightarrow h w \gamma p \delta v(h \in \mathscr{A} \delta v)$. Thus $(G F)(p) \sigma(\mathscr{A})(f)=f \delta v w \gamma p \delta v=f \delta w \gamma p \delta v=$. $f \delta \gamma \delta \delta=f \gamma p \delta v=f p \delta v$ since $v w=w, \delta w=\delta, \delta \gamma=\gamma$ and $f \gamma=f$. On the other hand $\sigma(\mathscr{B})(f p)=f p \delta v$.

For each $\mathscr{A}, \sigma(\mathscr{A})$ is an isomorphism, its inverse being $h \rightarrow h \omega \gamma(h \in \mathscr{A} \delta v)$. The proof is completed.

Let $N$ be a minimal subset of $\beta T, v \in N$ and $\mathscr{A}$ an object of $K(v)$. Then $\mathscr{A}$ induces a topology $\tau_{v}(\mathscr{A})$ on $N v$ just as in $\S 2$. Thus if $p \in N v$ and $A \subset N v$, then $p$ is in the $\tau_{v}(\mathscr{A})$ closure of $A$ if and only if $f p \leqq f^{A} v(f \in \mathscr{A})$.

6.4 Proposition. Let $N$ and $L$ be minimal subsets of $\beta T, v \in N \cap J, \delta, \gamma \in L \cap J$ with $v \gamma=v$ and $\gamma v=\gamma$. Then the mapping $\phi:\left(N v, \tau_{v}(\mathscr{A})\right) \rightarrow\left(L \delta, \tau_{\delta}(\mathscr{A} \delta)\right)$ such that $\phi(p)=\gamma p \delta$ is a homeomorphism and group isomorphism for all T-subalgebras $\mathscr{A}$ of $\mathfrak{A}(v)$, the inverse being the map $\psi$ such that $\psi(g)=v q \gamma(q \in L \delta)$.

Proof. Let $p, q \in N v$. Then $\phi(p q)=\gamma p q \delta=\gamma p \gamma q \delta=\gamma p \delta \gamma q \delta=\phi(p) \phi(q)$ since $p \gamma=p$ and $\delta \gamma=\gamma$. Also, $\psi \phi(p)=v \phi(p) \gamma=v \gamma p \delta \gamma=v p \gamma=p(p \in N v)$; and $\phi \psi(r)=\gamma \psi(r) \delta$ $=\gamma v r \gamma \delta=\gamma r \delta=r(r \in L \delta)$. Hence $\phi$ is a group isomorphism of $N v$ onto $L \delta$.

Now let $A \subset N v$ and $p \in N v$ with $p$ in the $\tau_{v}(\mathscr{A})$-closure of $A$ and let $f \in \mathscr{A} \delta$. Then $f=h \delta$ for some $h \in \mathscr{A}$, and $f \phi(p)=h \delta \gamma p \delta=h \gamma p \delta=h p \delta$. Now $h p \leqq h^{A} v=h^{A \gamma} v=(h \gamma)^{A \gamma} v$ $=(h \delta \gamma)^{A \gamma} v=(h \delta)^{\gamma A \gamma} v=f^{\gamma A \delta \gamma} v \leqq f^{\gamma A \delta} \gamma v=f^{\gamma A \delta} \gamma$. Hence $f \phi(p)=h p \delta \leqq f^{\gamma A \delta} \gamma \delta=f^{\phi(A)} \delta$. Thus $\phi(p)$ is in the $\tau_{\delta}(\mathscr{A} \delta)$-closure of $\phi(A)$. This proves that $\phi$ is continuous. The proof that $\psi$ is continuous is similar.

\section{REFERENCES}

1. N. Bourbaki, Eléments de mathématique, Livre VI, Chapitre III, Hermann, Paris, 1952.

2. Robert Ellis, Locally compact transformation groups, Duke Math. J. 24 (1957), 119-125.

3. - Distal transformation groups, Pacific J. Math. 8 (1958), 401-405.

4. - A semigroup associated with a transformation group, Trans. Amer. Math. Soc. 94 (1960), 272-281. 
5. Robert Ellis, Universal minimal sets, Proc. Amer. Math. Soc. 11 (1960), 540-543.

6. - Point transitive transformation groups, Trans. Amer. Math. Soc. 101 (1961), 384-395.

7. - Group-like extensions of minimal sets, Trans. Amer. Math. Soc. 127 (1967), 125-135.

8. H. Furstenberg, The structure of distal flows, Amer. J. Math. 85 (1963), 477-515.

9. W. H. Gottschalk and G. A. Hedlund, Topological dynamics, Amer. Math. Soc. Colloq. Publ., Vol. 36, Amer. Math. Soc., Providence, R. I., 1955.

10. B. Horelick, An algebraic theory of minimal sets, Thesis, Wesleyan Univ., Middletown, Conn., 1967.

11. A. W. Knapp, Distal functions, Proc. Nat. Acad. Sci. U.S.A. 52 (1964), 1409-1412.

12. - Distal functions on groups, Preprint, Massachusetts Institute of Technology, Cambridge, Mass., 1966.

WESLEYAN UNIVERSTTY, MIDDLETOWN, CONNECTICUT

EIDGENOESSISCHE TECHNISCHE HOCHSCHULE, ZUERICH, SWTTZERLAND 